\title{
Varying the Aspect Ratio of Toroidal Ion Traps: Implications for Design, Performance, and Miniaturization
}

Praneeth Madushan Hettikankanange

Brigham Young University

Follow this and additional works at: https://scholarsarchive.byu.edu/etd

Part of the Physical Sciences and Mathematics Commons

\section{BYU ScholarsArchive Citation}

Hettikankanange, Praneeth Madushan, "Varying the Aspect Ratio of Toroidal Ion Traps: Implications for Design, Performance, and Miniaturization" (2020). Theses and Dissertations. 8734.

https://scholarsarchive.byu.edu/etd/8734

This Thesis is brought to you for free and open access by BYU ScholarsArchive. It has been accepted for inclusion in Theses and Dissertations by an authorized administrator of BYU ScholarsArchive. For more information, please contact ellen_amatangelo@byu.edu. 
Varying the Aspect Ratio of Toroidal Ion Traps: Implications for Design, Performance, and Miniaturization

\title{
Praneeth M. Hettikankanange
}

\author{
A thesis submitted to the faculty of \\ Brigham Young University \\ in partial fulfillment of the requirements for the degree of \\ Master of Science \\ Daniel E. Austin, Chair \\ Jaron C. Hansen \\ Jeremy A. Johnson
}

Department of Chemistry and Biochemistry

Brigham Young University

Copyright (C) 2020 Praneeth M. Hettikankanange

All Rights Reserved 


\begin{abstract}
Varying the Aspect Ratio of Toroidal Ion Traps: Implications for Design, Performance, and Miniaturization

Praneeth M. Hettikankanange

Department of Chemistry and Biochemistry, BYU

Master of Science

A large aspect ratio leads to higher ion capacity in miniaturized ion trap mass spectrometers. The aspect ratio (AR) of an ion trap represents the ratio between an extended trapping dimension and the characteristic trapping dimension. In contrast to linear and rectilinear traps, changing the AR of a toroidal ion trap (TorIT) results in changes to the degree of curvature and shape of the trapping potential, and hence, on performance as a mass analyzer. SIMION simulations show that higher-order terms in the trapping potential vary strongly for small and moderate AR values (below 10 ), with the effects asymptotically flattening for larger AR values. Because of the asymmetry in electrode geometry, the trapping center does not coincide with the geometric center of the trap, and this displacement also varies with AR. For instance, in the asymmetric TorIT, the saddle point in the trapping potential and the geometric trap center differ from +0.6 to $-0.4 \mathrm{~mm}$ depending on AR. Ion secular frequencies also change with the AR. Whereas ions in the simplified TorIT have stable trajectories for any value of AR, ions in the asymmetric TorIT become unstable at large AR values. Variations in high-order terms, the trapping center, and secular frequencies with AR are a unique feature of toroidal traps, and require significant changes in trap design and operation as the aspect
\end{abstract} ratio is changed.

Keywords: toroidal ion Ttap, aspect ratio, saddle point, geometric center, higher-order multipoles, secular frequency 


\section{ACKNOWLEDGMENT}

I would like to convey my heartfelt gratitude towards Professor Daniel E. Austin for being an excellent and exceptionally insightful mentor throughout my graduate research career at his laboratory. He has been very patient during these three years and always encouraged me to be a worthy candidate to enter a vast research world. His advice, constant guidance, and tremendous mentorship have helped me to achieve the impossible. He has spent a significant amount of his valuable time helping my writing, refining, and composing research papers and my thesis dissertation.

I am grateful to all of those with whom I have had the pleasure to work during this and other related projects. Each of my thesis committee members has provided me extensive personal and professional guidance and taught me a great deal about scientific research and life in general. I would also like to thank my committee, professors Jaron C. Hansen, Ryan Kelly, and Jeremy A. Johnson. They have always been resourceful, encouraging, and patient even from very beginning of the program.

I would also like to thank my labmates in Austin's laboratory: Elaura Gustafson, Radhya Gamage, George Gao, Abraham De la Cruz, and Brandon Turner for their invaluable support in numerous insightful knowledge and constant support throughout the project.

I wish to acknowledge my family's support and great love, and words cannot express my gratitude for my wife, Ashari; my mother, Samanmali; and my sister, Iresha. They kept me going on and this work would not have been possible without their input in terms of constant support, understanding, and care they have expressed unconditionally and constantly even at difficult times. 


\section{TABLE OF CONTENTS}

COVER PAGE iv

ABSTRACT

.ii

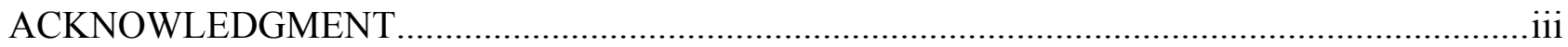

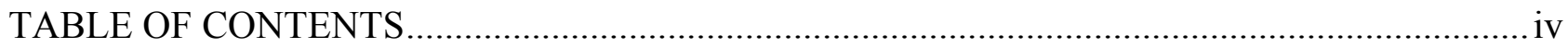

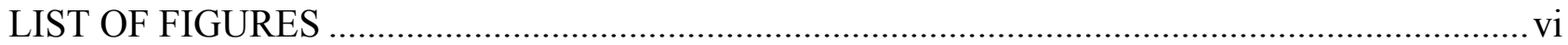

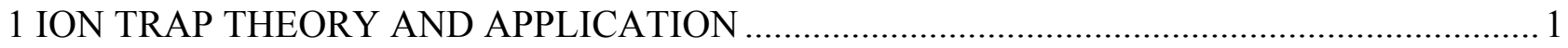

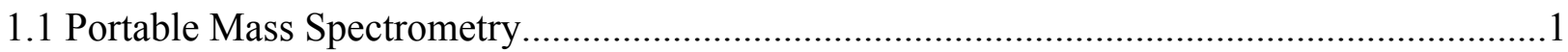

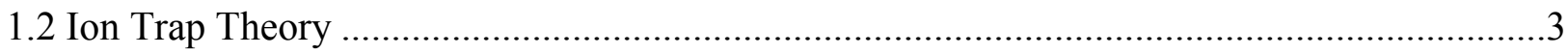

1.2.1 Quadrupole Ion trap Potential...................................................................................

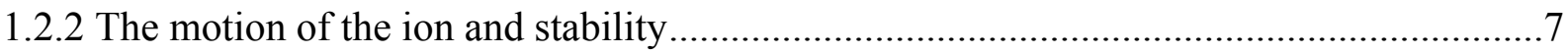

1.2.3 Non-Linear Electric Fields and Their Roles in Ion Traps ............................................12

1.2.4 Generalized Potential from Multipole Expansion ............................................................16

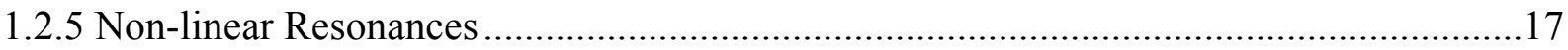

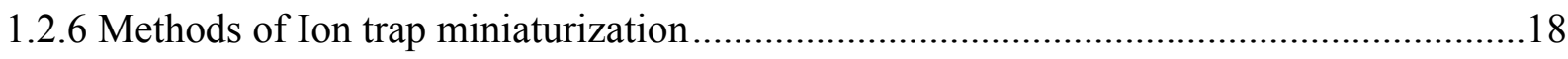

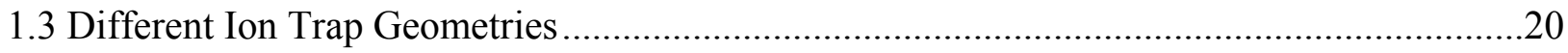

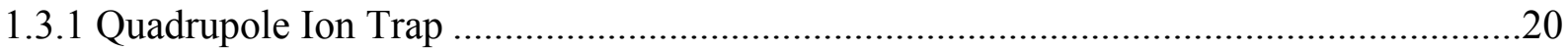

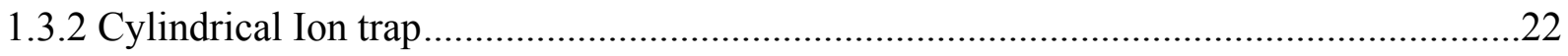

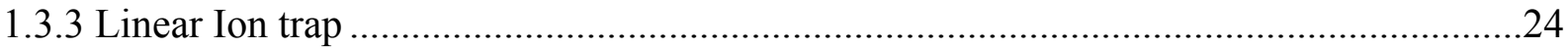

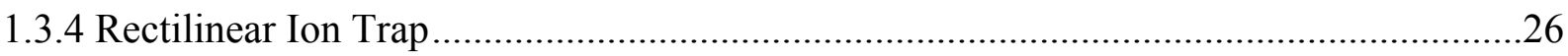


1.3.5 Toroidal Ion trap .28

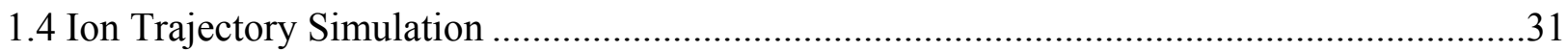

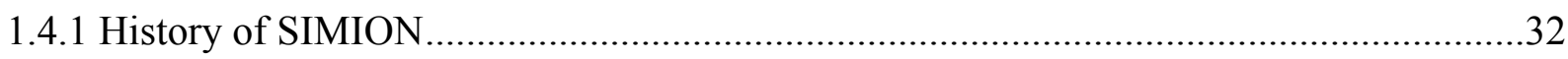

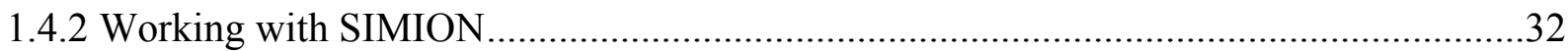

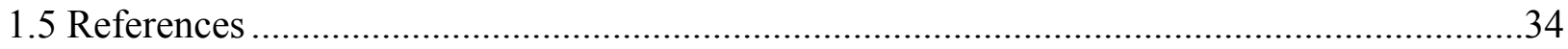

2 VARYING THE ASPECT RATIO OF TOROIDAL ION TRAPS: IMPLICATIONS FOR DESIGN, PERFORMANCE, AND MINIATURIZATION ……..................................................... 42

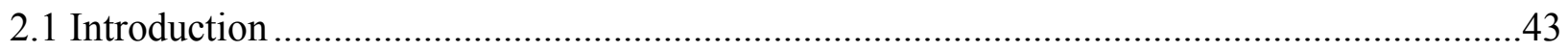

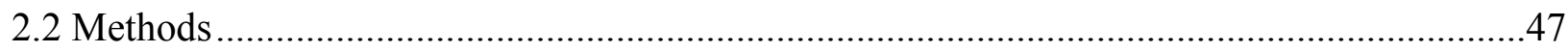

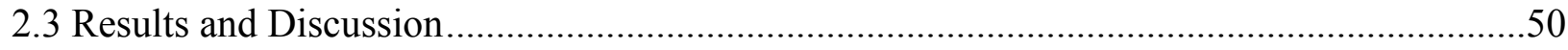

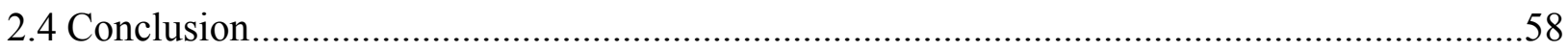

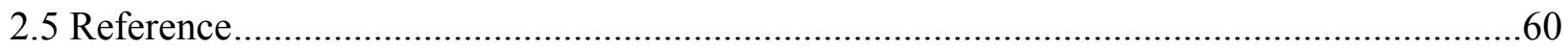

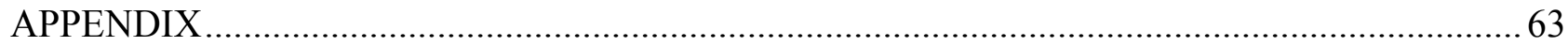

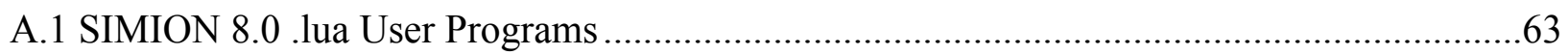

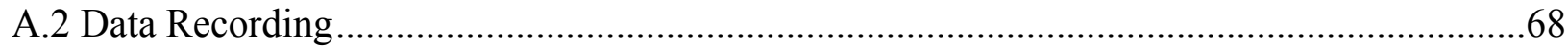

A.2 MATLAB Scripts for Data Processing ……………………………..............................68

A.2.1 MATLAB Script to Calculate Higher-Order Terms.........................................................68

A.2.3 MATLAB Script to generate frequency spectra............................................................69 


\section{LIST OF FIGURES}

Figure 1-1: Quadrupole ion trap; (a) photograph of an ion trap cut in half along the axis of cylindrical symmetry, (b) a schematic diagram of the three-dimensional ideal ion trap showing the asymptotes and the dimensions $r_{o}$ and $z_{o}$. (Adapted from R. E. March and J. F. J. Todd, Quadrupole Ion Trap Mass Spectrometry, Second Edition, Hoboken, NJ: Wiley-Inter Science, 2005)

Figure 1-2: Stability diagram in $\left(a_{z}, q_{z}\right)$ space in both $r$ and $z$ directions the near origin for the threedimensional QIT; the iso- $\beta_{r}$ and iso- $\beta_{z}$ lines are shown in the diagram. The $\mathrm{q}_{\mathrm{z}}$ axis intersects the $\beta_{z}=1$ boundary at $q_{z}=0.908$, which corresponds to $q_{\min }$ the mass-selective instability mode. (Reproduced from R. E. March and J. F. J. Todd, Quadrupole Ion Trap Mass Spectrometry, Second Edition, Hoboken, NJ: Wiley-Inter Science, 2005)

Figure1-3: Trajectory of a trapped ion of $\mathrm{m} / \mathrm{z} 105$. The initial position was selected randomly from a population with an initial Gaussian distribution (FWHM of $1 \mathrm{~mm}$ ); $q_{z}=0.3$; zero initial velocity. The projection onto the $\mathrm{x}-\mathrm{y}$ plane illustrates planar motion in three-dimensional space. The trajectory develops a shape that resembles a flattened boomerang. (Practical Aspects of Trapped Ion Mass Spectrometry, Volume IV: Theory and Instrumentation; March, R. E., Todd, J. F. J., Eds.; CRC Press: London, 2017)

Figure 1-4: The three electrodes of the quadrupole ion trap shown in open array. (Reproduced from March and Todd (2005, Fig. 2.11 of QITMS), copyright at 2005, with permission of John Wiley \& Sons, Ltd.)

Figure 1-5: (a) Concept and (b) experimental implementation of a cylindrical ion trap as an approximation to the hyperbolic Paul ion trap. (Adapted from Wells, J. M.; Badman, E. R.; Cooks, R. G. A Quadrupole Ion Trap with Cylindrical Geometry Operated in the Mass-Selective Instability Mode. Anal. Chem. 1998, 70 (3), 438-444)

Figure 1-6: Basic design of the two-dimensional linear ion trap. (Adapted from Schwartz, J. C.; Senko, M. W.; Syka, J. E. P. A Two-Dimensional Quadrupole Ion Trap Mass Spectrometer. J. Am. Soc. Mass Spectrom. 2002, 13 (6), 659-669)

Figure 1-7: Conceptual evolution of the rectilinear ion trap and interrelationship to other types of ion traps (Adapted from Snyder, D. T.; Pulliam, C. J.; Ouyang, Z.; Cooks, R. G. Miniature and Fieldable Mass Spectrometers: Recent Advances. Anal. Chem. 2016, 88 (1), 2-29)

Figure 1-8: Configuration of the rectilinear ion trap and the control signals applied to it. (Reproduced from Ouyang, Z.; Wu, G.; Song, Y.; Li, H.; Plass, W. R.; Cooks, R. G. Rectilinear Ion Trap: Concepts, Calculations, and Analytical Performance of a New Mass Analyzer. Anal. Chem. 2004, 76 (16), 4595-4605)

Figure 1-9: Comparison of the QIT and the toroidal ion trap. For the QIT, the rotational axis passes through the trapping center. (Adapted from Lammert, S. A.; Plass, W. R.; Thompson, C. V.; Wise, 
M. B. Design, Optimization and Initial Performance of a Toroidal Rf Ion Trap Mass Spectrometer. Int. J. Mass Spectrom. 2001, 212 (1-3), 25-40)

Figure 2- 1: Cross-section views of asymmetric TorIT (a) and simplified TorIT (b) with DC and RF electrodes. The dimensions for the asymmetric TorIT and simplified TorIT were taken from Lammert et al. [12] and Taylor and Austin [6], respectively. The aspect ratio (AR) of any TorIT is defined as the ratio between the annular radius (inscribed toroidal radius) $\left(R_{0}\right)$ to the trap

Figure 2-2: Effect of aspect ratio on the percent hexapole $\left[\left(\mathrm{A}_{3} / \mathrm{A}_{2}\right) \times 100 \%\right]$, octopole $\left[\left(\mathrm{A}_{4} / \mathrm{A}_{2}\right)\right.$ $\times 100 \%$, decapole $\left[\left(\mathrm{A}_{5} / \mathrm{A}_{2}\right) \times 100 \%\right]$, and dodecapole $\left[\left(\mathrm{A}_{6} / \mathrm{A}_{2}\right) \times 100 \%\right]$ in the simplified TorIT in radial direction (a) and asymmetric TorIT in axial direction (b). The trapping radius, $\mathrm{r}_{0}$, was held constant at $10 \mathrm{~mm}$ and $5.8 \mathrm{~mm}$ for asymmetric TorIT and simplified TorIT respectively. The last points represent the corresponding higher-order terms of LIT with the same electrode geometry.

Figure 2-3: Separation of the saddle point from the geometric trap center in the asymmetric TorIT and simplified TorIT with the aspect ratio.

Figure 2-4: r-z view of the Asymmetric TorIT illustrating the asymmetric Coulombic force on ions resulting in the trapping center being different from the geometric center. The inner electrode exerts strong Columbic force on the ion than the outer electrode due to the asymmetric cross section (a). $\mathrm{x}$ $y$ view of the TorIT with one ion in the ion cloud. The outer electrode exerts strong Columbic force on the ion due to the angular influence of inner and outer ring electrodes (b).

Figure 2-5: Effect of aspect ratio on the secular frequency of simplified and asymmetric TorITs for the ion of $100 \mathrm{~m} / \mathrm{z}$. The conditions applied to the trap was $500 \mathrm{~V}_{0-\mathrm{p}}$ r.f voltage with no DC offset under $1 \mathrm{MHz}$ r.f. frequency with no collision gas. The last points represent the corresponding secular frequencies of LIT with the corresponding electrode geometry.

Figure 2-6: Ion ejection efficiency of simplified TorIT in radial direction. AC voltage applied on the endcap electrode $\left(V_{0-p}=2 \mathrm{~V}\right)$ to excite the ion with $\mathrm{m} / \mathrm{z}$ of 100 via dipolar resonant ejection. The frequency for the $\mathrm{AC}$ voltage was set to a value that is few percent lower the exact secular frequency of the ion. This auxiliary AC frequency was determined experimentally by selecting the maximum frequency that can eject the ions. The hard sphere collision cooling technique was incorporated under the pressure and temperature of $1.5 \mathrm{mTorr}$ and $273 \mathrm{~K}$ respectively. 


\section{ION TRAP THEORY AND APPLICATION}

\subsection{Portable Mass Spectrometry}

One principal characteristic of analytical chemistry is identifying and quantifying atomic or molecular species present in a given system. Mass spectrometry (MS) is arguably the most critical analytical spectroscopic technique for qualitative and quantitative characterization of chemical species analysis [1]. Since the early $20^{\text {th }}$ century, it has been widely used due to its high sensitivity, precision, and compatibility with chromatography [2]. The mass spectrometry process consists of several significant steps, including producing ions in the gaseous phase, fragmentation and separation of ions based on the mass-to-charge ratio $(\mathrm{m} / \mathrm{z})$, and finally detection of ions.

The ability to make real-time in situ chemical measurements of a given system in its original state has become more essential to researchers in nearly all science and engineering fields. Conventionally, a sample is collected from the source of interest and sent to the laboratory for analysis on a benchtop instrument. However, the procedures involved in such a process make unfavorable circumstances and conditions. Also, for instances such as the solar system where returning samples is very difficult. There is also some motivation to make mass spectrometers (MS) less expensive so they can be more widely used. To address such unfavorable situations, scientists may require a mass spectrometer that is compact, lightweight, robust, sensitive, versatile, reliable, cost-effective, and

field-portable. Such field-portable mass spectrometers with relatively good analytical performance and ability of continuous analyzing are the ideal features for a wide variety of situ applications. 
One method of separating ions with different mass/charge $(\mathrm{m} / \mathrm{z})$ is through analysis in a dynamic radiofrequency (r.f.) electric field. Such mass analyzers are referred to as ion trap mass analyzers. The trapped ions make stable or unstable trajectories based on the ion trap dimensions, conditions applied to the ion trap, and $\mathrm{m} / \mathrm{z}$ ratio. Depending on the ion trap and electrodes' geometry, various ion trap designs have been developed to improve either performance or manufacturability.

A good mass spectrometry system usually satisfies three basic requirements: portability, high specificity, and sensitivity. The most effective way to introduce a user-friendly portable mass spectrometer is to reduce the size of the mass analyzer. Ion trap mass analyzes have become the most common type of MS used today due to their relatively small size, lightweight, low-cost, and ability to miniaturize compared to sector-type mass analyzers. The miniaturization of mass analyzers allows ions to have a smaller mean free path (MFP). The smaller MFP makes more favorable conditions to operate the mass analyzer at higher operating pressure with the help of a smaller pump. Also, the reduced dimensions of the mass analyzer demand a smaller detector, a vacuum chamber, and a reduced gas load for a lower-power pump. The r.f. trapping voltage is proportional to the square of the mass analyzer's trapping radius, which causes a modest reduction in mass analyzer size and allows us to operate the mass analyzer at a lower operating voltage. A portable battery system supplies the power requirement of the ion trap mass analyzer. However, miniaturization of ions traps encounters specific challenges that could limit the degree of miniaturization. One of the main challenges is the space charge effect, as it determines the ion storage capacity. Since miniaturized ion traps have smaller trapping volumes, they can hold only a limited amount of ions. On the other hand, a lower tolerable charger density exists in an ion trap as a result of the lower r.f. voltage on electrodes. So, 
ion storage capacity is mainly limited by reductions in both charge density and the trapping volume. The result of the lower charge density and trapping volume is poor sensitivity.

Various versions of full-size ion trap mass analyzers are commercially available. Each version of mass analyzer has unique advantages and disadvantages. Also, several portable versions of ion trap mass spectrometers have been developed and are commercially available including Mini10 [3], Mini11 [4], and Mini12 [5] from Purdue University, Torion T-9 GC-MS from PerkinElmer [6], A Miniaturized System of a Gas Chromatograph Coupled with a Paul Ion Trap Mass Spectrometer from the Jet Propulsion Laboratory [7], Chemsense600 [8] from Griffin Analytical Technology LLC., and the MMS-1000 from $1^{\text {st }}$ Detect Inc. Since the mass analyzer is the main feature that determines the analytical performance of the analysis, much more attention and effort have been given to optimizing the mass analyzer when designing at reduced dimensions. Depending on the geometry of the ion trap and geometry of the electrodes, various ion trap designs have been developed to improve either performance or manufacturability. These geometries have unique capabilities because the electric field shape is defined and constrained by the electrode shape.

\subsection{Ion Trap Theory}

In 1953, Wolfgang Paul and collaborators developed a new technique for storing and manipulating ions by alternating r.f. electric fields [9]. Their methodologies utilized quadrupole fields, and the design was based on taking the cross-section of the quadrupole mass filter (QMF) and rotate it around the central axis. The initial electrode shape was hyperbolic and had an asymptotic slope. In the early 1960s, Paul and his collaborators successfully demonstrated the trapping capability for mass analysis [32]. 


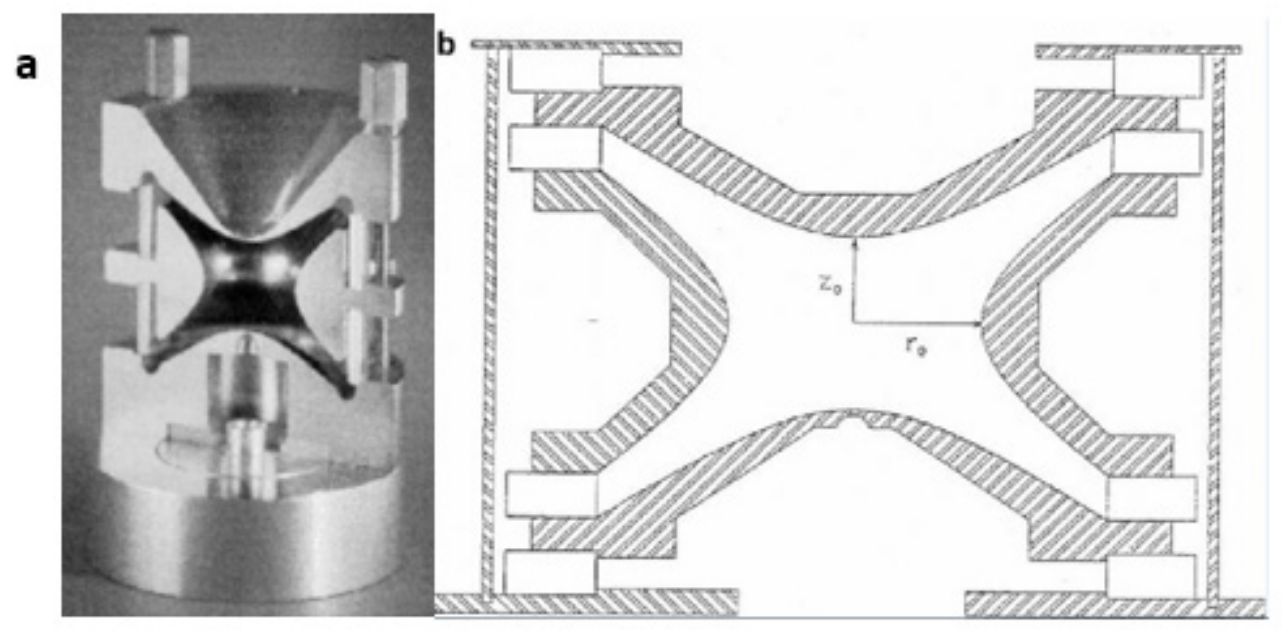

Figure 1-1: Quadrupole ion trap; (a) photograph of an ion trap cut in half along the axis of cylindrical symmetry, (b) a schematic diagram of the three-dimensional ideal ion trap showing the asymptotes and the dimensions $r_{o}$ and $z_{0}$. (Adapted from R. E. March and J. F. J. Todd, Quadrupole Ion Trap Mass Spectrometry, Second Edition, Hoboken, NJ: Wiley-Inter Science, 2005)

\subsubsection{Quadrupole Ion trap Potential}

In their approach, Paul and his collaborators utilized quadrupolar electric fields, which has potentials that scale with the square of $\mathrm{x}, \mathrm{y}$, and $\mathrm{z}$ in the Cartesian coordinate system:

All the equations from 1-1 to 1- 37 are taken from reference [10].

$\phi(x, y, z)=A\left(\lambda x^{2}+\sigma y^{2}+\gamma z^{2}\right)+C$

Where, $\mathrm{x}, \mathrm{y}$, and $\mathrm{z}$ are the rectangular coordinates at a given point, $\mathrm{A}$ is the electric potential (r.f. voltage) applied on the ring electrode and C is a "fixed" (which also may be an r.f. potential either alone or combination with a DC potential) potential applied to all the electrode electrodes, and $\lambda, \sigma$, and $\gamma$ are weighting constants for their respective coordinates. 
Equation 1 should satisfy the condition of the Laplace equation, which states that the sum of the second partial derivatives of quadruple potential inside the trap should be zero, i.e

$\nabla^{2} \phi(x, y, z)=0$ or $\nabla^{2} \phi(\mathrm{x}, \mathrm{y}, \mathrm{z})=\frac{\partial \phi(\mathrm{x}, \mathrm{y}, \mathrm{z})}{\partial \mathrm{x}}+\frac{\partial \phi(\mathrm{x}, \mathrm{y}, \mathrm{z})}{\partial \mathrm{y}}+\frac{\partial \phi(\mathrm{x}, \mathrm{y}, \mathrm{z})}{\partial \mathrm{z}}$

Taking the partial derivatives of the Eqn (1-2) gives,

$\nabla^{2} \phi(x, y, z)=2 A(\lambda+\sigma+\gamma)=0$

Solving the equation 1-3,

Since $A \neq 0$

$(\lambda+\sigma+\gamma)=0$

This equation can be satisfied in an infinite number of combinations, with different values for the coefficients $\lambda, \sigma$, and $\gamma$. One of the most well-known solutions utilizes the combination of $\lambda=1$, $\sigma=1$ and $\gamma=-2$. (because the QIT is cylindrically symmetric). So, the potential of the equation is given by

$\phi(x, y, z)=A\left(x^{2}+y^{2}-2 z^{2}\right)+C$

If this potential is instead expressed in polar coordinates, it takes the form:

$\phi(\mathrm{r}, \mathrm{z})=\mathrm{A}\left(\mathrm{r}^{2}-2 \mathrm{z}^{2}\right)+\mathrm{C}$ 
This potential is generated using three rotationally symmetric electrodes. They have a hyperbolic geometric shape. The center electrode is most often referred to as the "ring" electrode. It has the general form:

$\frac{\mathrm{r}^{2}}{\mathrm{r}_{0}^{2}}-\frac{2 \mathrm{z}^{2}}{\mathrm{r}_{0}^{2}}=1$

The other two electrodes which are placed above and below the ring electrodes are called end caps electrodes. The endcap electrodes also take the hyperbolic shape.

$\frac{r^{2}}{2 z_{0}^{2}}-\frac{z^{2}}{z_{0}^{2}}=-1$

Both ring electrode and endcap electrodes induce the potential at any point inside the quadrupole ion trap. Therefore, the net potential, $\phi_{0}$, is the difference of the potentials applied on ring electrode and endcap electrodes.

$\phi_{0}=\phi_{\text {ring }}-\phi_{\text {endcaps }}$

By applying the boundary conditions, the coefficient of A and C can be found,

At $z=0$ and $r=r_{0}, \phi_{0}=\phi_{\text {ring }}$

At $r=0$ and $r=z_{0}, \phi_{0}=\phi_{\text {endcap }}$

By solving the potential equation at boundary conditions, the coefficient of $\mathrm{A}$ and $\mathrm{C}$ are,

$A=\frac{\phi_{0}}{\left(\mathrm{r}_{0}^{2}+2 \mathrm{z}_{0}^{2}\right)}$ 
$C=\frac{2 \phi_{0} \mathrm{z}_{0}^{2}}{\mathrm{r}_{0}^{2}+2 \mathrm{z}_{0}^{2}}$

The potential at any point inside the trap can be determined using,

$\phi(r, z)=\frac{\phi_{0}}{\left(\mathrm{r}_{0}^{2}+2 \mathrm{z}_{0}^{2}\right)}\left(\mathrm{r}^{2}-2 \mathrm{z}^{2}\right)+\frac{2 \phi_{0} \mathrm{z}_{0}^{2}}{\mathrm{r}_{0}^{2}+2 \mathrm{z}_{0}^{2}}$

Where,

$\phi_{0}=U+V \cos \Omega t$

Combining all the equations, combined with the general form of the quadrupole potential (expressed in cylindrical coordinates) provides:

$\phi(r, z)=\frac{(\mathrm{U}+\mathrm{V} \cos \Omega \mathrm{t})}{\left(\mathrm{r}_{0}^{2}+2 \mathrm{z}_{0}^{2}\right)}\left(\mathrm{r}^{2}-2 \mathrm{z}^{2}\right)+\frac{2(\mathrm{U}+\mathrm{V} \cos \Omega \mathrm{t}) \mathrm{z}_{0}^{2}}{\mathrm{r}_{0}^{2}+2 \mathrm{z}_{0}^{2}}$

1.2.2 The motion of the ion and stability

Consider the motion of an ion in the $\mathrm{z}$-direction, specifically on the axial direction $(\mathrm{r}=0$ axis). The force the ion experiences can be determined by the electric field, which is the first derivative of the potential with respect to z:

The electrostatic force that exerts on the ion can be determined by,

$F_{z}=-e E_{z}$

The electric field is the first derivative of the electric potential, 
$E=\frac{\partial \phi}{\partial z}$

The force on the ion in the electric field can also be calculated by using the Newtonian equation,

$F_{z}=m a_{z}$

$F_{z}=-e \frac{\partial \phi}{\partial z}$

$F_{Z}=\frac{4 e(U+V \cos \Omega t) z}{r_{0}^{2}+2 z_{0}^{2}}$

$F_{z}=m a=m \frac{\partial^{2} z}{\partial^{2} t}=\left(\frac{4 e U}{r_{0}^{2}+2 z_{0}^{2}}+\frac{4 e V \cos \Omega t}{r_{0}^{2}+2 z_{0}^{2}}\right) Z$

The equation can be slightly rearranged to provide:

$\frac{\partial^{2} z}{\partial^{2} t}=\left(\frac{4 e U}{m\left(r_{0}^{2}+2 z_{0}^{2}\right)}+\frac{4 e V \cos \Omega t}{\left(r_{0}^{2}+2 z_{0}^{2}\right)}\right) Z$

The electric potential applied to the electrodes creates a force on the ion. The Mathieu equation can describe the ion motion that results from this force

$\frac{\partial^{2} u}{d \xi^{2}}+\left(a_{u}-2 q_{u} \cos 2 \xi\right) u=0$

Where $u$ represents either $\mathrm{x}, \mathrm{y}$, or $\mathrm{z}$ and $\xi=\frac{\Omega}{2}$. Also, to fit the second-order partial derivative equation to the format of the Mathieu equation, the Mathieu stability parameters, $a_{u}$ and $q_{u}$ can be defined as

$a_{z}=\frac{-16 e U}{m\left(r_{0}^{2}+2 z_{0}^{2} \Omega^{2}\right.}$ 
$q_{z}=\frac{8 e U}{m\left(r_{0}^{2}+2 z_{0}^{2} \Omega^{2}\right.}$

Mathieu's equation has two types of solutions. The first type of solution is periodic and "stable" in which the amplitude of the ion does not increase to a large amplitude with time. The second condition is periodic, but "unstable" means is that amplitude does increase to infinitely large values over time. Any ion that meets the conditions to be outside of the ion trap will not remain trapped inside the quadrupole ion trap. It moves away from the center of the trap with time and eventually hits an electrode or leaves the trap through the exit slits. However, if an ion satisfies the stability condition, it remains confined in the trap. In order to understand the conditions in which the ion trap is operating, it is essential to know if an ion can be successfully trapped or not. It is critical to consider the values of $\beta_{u}$ for both the $\mathrm{r}$ and $\mathrm{z}$ dimensions to evaluate if the ion makes a stable or unstable motion. The parameter $\beta_{u}$ is defined as and can be calculated by the recursion formula

$$
\beta_{u}^{2}=a_{u}+\frac{q_{u}^{2}}{\left(\beta_{u}+2\right)^{2}-a_{u}-\frac{q_{u}^{2}}{\left(\beta_{u}+4\right)^{2}-a_{u}-\frac{q_{u}^{2}}{\left(\beta_{u}+6\right)^{2}-a_{u}-\cdots}}}+\frac{q_{u}^{2}}{\left(\beta_{u}-2\right)^{2}-a_{u}-\frac{q_{u}^{2}}{\left(\beta_{u}-4\right)^{2}-a_{u}-\frac{q_{u}^{2}}{\left(\beta_{u}-6\right)^{2}-a_{u}-\cdots}}}
$$

For any values for $\beta_{u}$ between 0 to 1 , the ion makes a stable trajectory in the trap. It also implies that an ion's motion can be considered stable when both $\beta_{r}$ and $\beta_{z}$ are between 0 and 1.0. If the ion's stability is outside this range for $\beta_{r}$, the ion is ejected radially. Also, any value for $\beta_{z}$ outside the stability range, the ion is ejected axially. This mathematical formula can be represented graphically using a stability diagram for the trap. 


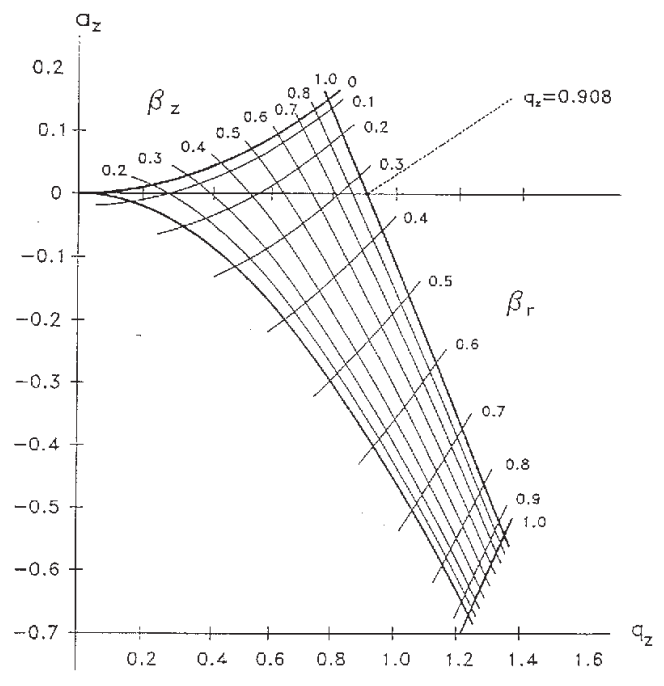

Figure 1-2: Stability diagram in $\left(a_{z}, q_{z}\right)$ space in both $r$ and $z$ directions the near origin for the threedimensional QIT; the iso- $\beta_{r}$ and iso- $\beta_{z}$ lines are shown in the diagram. The $q_{z}$ axis intersects the $\beta z=1$ boundary at $q z=0.908$, which corresponds to $q_{\min }$ the mass-selective instability mode. (Reproduced from R. E. March and J. F. J. Todd, Quadrupole Ion Trap Mass Spectrometry, Second Edition, Hoboken, NJ: Wiley-Inter Science, 2005)

The motion of the ion within the quadrupole ion trap is quite complicated as ions move in 3D space and the motion of an ion is studied separately in each direction. The fundamental frequency at which the ion moves in all directions within the trap is called the ion's fundamental secular frequency. The two main components that compose the fundamental secular frequency in radial and axial directions are $\omega_{r}$ and $\omega_{z}$ respectively. The following equations mathematically describe this. 


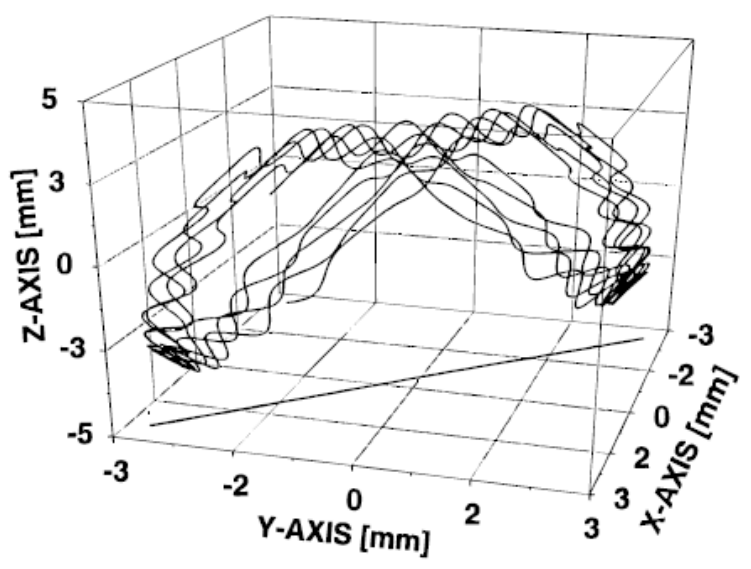

Figure1-3: Trajectory of a trapped ion of $\mathrm{m} / \mathrm{z} 105$. The initial position was selected randomly from a population with an initial Gaussian distribution (FWHM of $1 \mathrm{~mm}$ ); $q_{z}=0.3$; zero initial velocity. The projection onto the $\mathrm{x}-\mathrm{y}$ plane illustrates planar motion in three-dimensional space. The trajectory develops a shape that resembles a flattened boomerang. (Practical Aspects of Trapped Ion Mass Spectrometry, Volume IV: Theory and Instrumentation; March, R. E., Todd, J. F. J., Eds.; CRC Press: London, 2017.)

$$
\begin{aligned}
& \omega_{u, n}=\left(n+\frac{1}{2} \beta_{u}\right) \Omega \quad 0 \leq n<\infty \\
& \omega_{u, n}=-\left(n+\frac{1}{2} \beta_{u}\right) \Omega \quad-\infty<n<0
\end{aligned}
$$

Where,

$\beta_{u} \approx \sqrt{\left(a_{u}+\frac{q_{u}^{2}}{2}\right)}$ at very low $a_{u}$ and $q_{u}$ values.

And also, considering only the axial component, $\beta_{z}$ can be approximated to

$\beta_{z \approx \sqrt{\frac{q_{z}^{2}}{2}}}$

And corresponding secular frequency can be defined as

$\omega_{z}=\frac{\beta_{z} \Omega}{2}$ 
$n$ can be an integer that ranges from zero to $\pm \infty$. There are a large number of secular frequencies in any given ion's motion. However, each secular frequency's relative strength drops very quickly from $n=0$ to $n= \pm 1, n= \pm 2$, etc. Since the "fundamental" $(n=0)$ frequency is the most substantial component of a given ion's motion, the term "secular frequency" is typically used to describe the frequency at $\mathrm{n}=0$ alone.

In the early 1980s, a team of scientists, including George Stafford at Finnigan, developed an important technique of operating the QIT, which they named "mass selective instability mode" [10]. Before this was discovered, the most common way of isolating a single $\mathrm{m} / \mathrm{z}$ was to isolate the ion just under the apex of the stability diagram [11]. In the mass selective instability technique, the ions are pushed to the right of the stability diagram by the linear ramp of the r.f. amplitude. As a result, the ions cross over the stability boundary at $q_{z}=0.908$ in order of increasing $\mathrm{m} / \mathrm{z}$. The ions become unstable in the $\mathrm{z}$-direction and can leave the trap through the holes in the endcap electrodes.

\subsubsection{Non-Linear Electric Fields and Their Roles in Ion Traps}

Although the Mathieu theory discussed so far is an accurate description of ion motion in a quadrupole potential, it is essential to realize that an ideal potential cannot be created. The electric field inside the ion trap always has contributions from higher-order fields (e.g., hexapole, octopole, decapole, and dodecapole being the prominent members) for several reasons. In real ion traps, it is physically impossible to generate a pure quadrupole field. For example, some practical limitations generate non-quadrupolar field components along with the quadrupole field. The superimposed nonlinear electric fields are caused due to many reasons including electrodes that do not extend to infinity, holes in endcaps for ion entrance/exit [12-15], and imperfect machining of electrodes [16, 17]. 
Furthermore, misalignments of electrodes from the standard measurements can also introduce nonlinear fields at any stage during fabrication, assembly, and operation [15, 18-22]. Depending on the sizes of the electrodes, the nature of the electrode's geometry, and the fabrication method used, electrode surface roughness can become non-negligible and affect the analytical performance of the mass analyzer. We can determine the distribution of non-linear electric fields along a given direction and their magnitude by realizing that the actual field can be modeled as a sum of various multipole fields, including the sum of quadrupole and hexapole octopole, decapole, etc. [1].

The presence of higher-order contribution in r.f. potential makes the field non-linear in both $r$ and $\mathrm{z}$ directions of the cylindrical coordinate system. The field dependency on $\mathrm{r}$ and $\mathrm{z}$ coordinates can be seen in the first partial derivative of equation (1-33). In a pure quadrupole field, the ion trajectories in $\mathrm{r}$ - and $\mathrm{z}$ - directions of an ion are entirely independent of the amplitude and decouple to each other. The superposition of higher-order terms on a pure quadrupole produces a field that is no longer linear. As a result, ion motion in the $\mathrm{r}$ and $\mathrm{z}$ dimensions cannot be treated independently, and this coupling allows energy to be exchanged between radial and axial direction. Furthermore, these phenomena make the secular frequencies of the motion in both perpendicular directions dependent upon the amplitude of the motion [10]. Mathematical evidence of this can be found in the equations (1-33), showing the existence of cross-terms of the form rz. Due to the dependency of the secular frequency on amplitude, the ion experiences overtones in the frequencies that make up its motion. The ion takes up energy from the r.f. drive field and increase its amplitude of the secular oscillation and the ion shifts from the resonance. This phenomenon causes the ion to make a beat motion [23, 24]. Also, several types of non-linear resonance conditions exist for each type of multipole superposition, making resonance lines within the stability diagram's stability region [23, 24]. At the 
center of the trap, the ion experiences a perfectly linear field. However, as the ion moves away from the center along the $r$ or z-directions, the ion motion is affected by the other non-linear contributions.

The superimposed higher-order multipoles with the quadrupole fields can be either constructive or destructive, depending on the multipole's sign and nature (odd or even) [25]. Positive even multipoles are considered to be those that increase the electric field strength along the z-axis (in other words, increase more than linearity), while the negative even multipoles have the opposite effect. Lower-order terms can make a more significant influence at the trapping center than higherorder terms. In other words, higher-order terms have a weaker contribution at the center of the trap due to the higher powers in their spatial expression. As the ions move further from the trapping center, the fringes of the trapping volume become dominant, and thus the higher-order terms easily dominate the field. The pure forms of higher order fields are used as ion guides, in which the larger drift zone is advantageous for the simultaneous transmission of a broad mass range of ions, but at the cost of focusing ability [26, 27]. There is no published evidence that those pure higher-order fields have the capability of mass analysis $[28,29]$. The oscillation frequency of an ion increases with its amplitude when the non-linear fields reinforce the quadrupole electric field. The opposite effect is observed when the non-linear fields degrade the quadrupole field. For example, positive even multipoles and negative even multipoles reinforce and degrade the quadrupole field, respectively. As a result, positive even multipoles shift up the fundamental secular frequency, while negative even multipoles shift it down. In the case of odd multipoles contribution, the net effect is low because, during each oscillation cycle of the ion, the frequency is shifted alternately in both directions [23]. This phenomenon explains why the octopole and other even multipoles stabilize ions against resonant losses. For example, they interfere with the continual uptake of energy by shifting the ion off- 
resonance before it has been excited enough to leave the trap. Ions are further stabilized by the greater restoring forces of the higher-order terms that steepen the pseudopotential well at the edges of the trap, which causes to have to prolong storage times [30].

The behavior of the frequency shift helps to prevent loss of ions in ion traps. However, it also results in poor mass resolution [23]. For example, higher-order fields delay the ion ejection when the ion trap is operating under mass selective instability with negative even multipoles [23]. After crossing the stability region boundary and gaining amplitude, a shift to lower frequency momentarily returns the ion to stable conditions, establishing a beat oscillation that increases the temporal line width [10]. However, positive even multipoles instead accelerate the ion into unstable conditions and can thus be used to compensate for negative fields and significantly improve resolution. Higher-order terms can also create resonant conditions where ion motion can absorb energy from the trapping field at specific frequencies despite lying within a stable region of the stability diagram [31].

Another effect of higher-order terms includes the generation of a resonant condition in which an ion's motion can absorb energy from the trapping field at particular frequencies regardless of where it is sitting within the stable region of the stability diagram. This phenomenon is referred to as the non-linear resonance effect. 


\subsubsection{Generalized Potential from Multipole Expansion}

The equation below shows the potential inside a real quadrupole ion trap mass spectrometer.

$\Phi(r, z)=\Phi_{0} \sum_{n=0}^{\infty} A_{n} \emptyset_{n}(r, z)$

Where $n$ represents the order of each multipole; for example, $(\mathrm{n}=0)$ monopole, $(\mathrm{n}=1)$ dipole, $(\mathrm{n}=2)$ quadrupole, $(n=3)$ hexapole, $(n=4)$ octapole etc. These terms can also be categorized as odd terms $\left(\mathrm{n}=1, \mathrm{n}=3, \mathrm{n}=5, \mathrm{n}=7 \ldots\right.$ etc) or even terms $(\mathrm{n}=2,2=4, \mathrm{n}=6, \mathrm{n}=8)$. The coefficient $A_{n}$ is a weighting factor for each multipole [10]; the small number for the value $A_{n}$ means the decreased magnitude of higher-order multipole contribution to the overall linear field inside the trap. The net potential, $\emptyset_{n}(r, z)$, is a function of specific field distribution associated with each multipole. This field distribution can be calculated using the following equation.

$\phi_{N}(\rho, \theta, \phi)=\Phi_{0} \sum_{n=0}^{\infty} A_{n} \frac{\rho^{2}}{r_{o}^{n}} p^{n}(\cos \theta)$

Where $P_{n}$ is the Legendre polynomial of order $\mathrm{n}$, and $\theta, \rho$ are the polar coordinates.

In this equation, we consider that potential is independent of coordinate $\phi$ because of the rotational symmetry.

Solving and rearranging the above two equations can be presented as

$\Phi_{r, z}=\Phi_{0}\left(A_{2} \frac{r^{2}-2 z^{2}}{2 r_{0}^{2}}+A_{3} \frac{3 r^{2} z-2 z^{3}}{2 r_{0}^{3}}+A_{4} \frac{3 r^{4}-24 r^{2} z^{2}+8 z^{4}}{8 r_{0}^{4}}+A_{5} \frac{15 r^{4} z-40 r^{2} z^{3}+8 z^{5}}{8 r_{0}^{5}}+\right.$

$A_{6} \frac{5 r^{6}-90 r^{4} z^{2}+120 r^{4} z^{2}-16 z^{6}}{16 r_{0}^{6}}$ 


\subsubsection{Non-linear Resonances}

In a pure quadrupole ion trap, the stability mainly depends on only the $q_{u}$ and $a_{u}$ Mathieu parameters. But in the case of a real trap, non-linear fields have cross-dimensional products in their expression, which bring complexity to the equation of motion such that there are no known analytical solutions. However, with a weak superposition of higher terms in an ion trap, the derived QIT theory can be redesigned by considering the additional fields as perturbations split from the linear portion. Wang et al. have derived a set of rules required to make resonance conditions and predictions for their relative strengths. To summarize the results, a resonance condition arises within the stable region bounded by $\beta_{r}>0$ and $\beta_{z}<1$ when the denominator of a specific expression becomes 0 , which in simplified form is:

$n_{r} \beta_{r}+n_{z} \beta_{z}=2 v$

Where $n_{r}, n_{z}$ and $v$ are integer values. Using the relationship between the $\beta_{u}$ terms to the fundamental secular frequencies defined in Eqn. 1-27:

$\omega_{u}=\beta_{u} \Omega / 2$

This condition can be rewritten as:

$n_{r} \omega_{r}+n_{z} \omega_{z}=v \Omega$

The physical meaning of the above equation is that resonances are produced when linear combinations of secular frequency harmonics meet a harmonic drive frequency. 
Various restrictions on $n_{r}, n_{z}$ and $v$ yield different types of resonances. For $v=0$, only coupling resonances can be generated where energy is simply exchanged between the $r$ and $z$ oscillations. Resonances can pick up energy from the r.f. drive when $v=1$. This condition includes the onedimensional axial resonances where $n_{r}=0$ and the radial resonances where $n_{z}=0$, as well as sum resonances where $n_{r}$ and $n_{z}$ have the same sign and both directions are energized simultaneously. A difference of resonances (where $n_{z}$ and $n_{r}$ opposite signs) results in energy exchange between $r$-and $z$-directions of motion and less energy are picked up from the field.

Based on rotational symmetry, $n_{r}$ must be even for all multipoles, while $n_{z}$ must be even only for even multipoles. The order $N$ of resonance can be defined as;

$N=\left|\mathrm{n}_{\mathrm{r}}\right|+\left|\mathrm{n}_{\mathrm{z}}\right|$

The resonance scan exists for values of $N, N-2, N-4$, etc., with a maximum of $2 N$. The strongest resonances for a given multipole are equivalent order such that $N=n$; furthermore, across different multipoles, those of lowest orders are predicted to have the most intense resonances.

\subsubsection{Methods of Ion trap miniaturization}

Although the mass analyzer is only a part of the overall mass spectrometer system, the size of the mass analyzer could determine the size of the overall system. For example, miniaturized mass analyzers have the capability of operating at higher pressures and lower voltages, resulting in application of lighter vacuum and power systems. Thus, it is important to consider giving attention to the size of the mass analyzer. 
The mathematical relationship with the stability coefficient shows in the following equations how the dimensions of the ion trap affect the stability of trapped ions.

$a_{z}=\frac{-16 e U}{m\left(r_{0}^{2}+2 z_{0}^{2}\right) \Omega^{2}}$

$q_{z}=\frac{8 e V}{m\left(r_{0}^{2}+2 z_{0}^{2}\right) \Omega^{2}}$

According to the above equations, the stability of an ion is controlled by the radial and axial dimensions of the trap $\left(r_{0}\right.$ and $\left.z_{0}\right)$, the magnitude of the r.f. and DC voltage ( $V$ and $U$ respectively in the equations), and r.f. frequency $(\Omega)$. Maintaining the stability of an ion in a trap with reduced dimensions can be done by maintaining suitably reduced r.f. and DC voltages or higher r.f. frequency or both. Reducing the dimensions and operating conditions in an ion trap brings certain benefits and drawbacks. Lower voltage can be supplied to the trap with the help of the electronics that consume lower power. Also, operating the trap at higher frequencies results in faster mass analysis time.

For an ion trap with reduced dimensions, the conventional vacuum systems are often inappropriate, either because of size and weight or mechanical considerations. Most of the small pumps that are commercially available cannot achieve a minimum pressure comparable to that of a laboratory roughing pump. Thus, the operation of the ion trap mass spectrometer at a relatively higher pressure than customary pressures would be desirable. Higher pressure operations can also result in higher sensitivity because of the greater sample concentration in the ionization region. However, the mass resolution suffers at higher operating pressures. Reducing ion trap dimensions creates limitations in the ion storage capacity and space charge effect. When ions are confined to a small space, their Coulombic charges cause repulsion and limit the number of ions that can be stored. More 
importantly, the Coulombic repulsion between ions distorts the local electric field, resulting in lower mass resolution, accuracy, sensitivity, and dynamic range. Additionally, manufacturing ion traps with reduced dimensions are difficult to do in millimeter-scale with acceptable tolerances. A tiny deviation in the submillimeter scale of the instruments results in the induction of superimposed higher-order fields. The manufacturing process has to be more accurate and precise for the smaller ion traps in order to get the given level of accuracy. As a result, there is a demand for high-quality microfabrication processes. Significant efforts have been made in miniaturization using microfabrication, including the microelectromechanical systems (MEMS) technique. This widely used technique is mainly based on semiconductor microfabrication processes. However, to overcome all these limitations, researchers have developed other ion trap designs that have increased ion capacity and/or electrode shapes for easier manufacturing.

\subsection{Different Ion Trap Geometries}

\subsubsection{Quadrupole Ion Trap}

The quadrupole ion trap is an extraordinary device that functions both as an ion store in which gaseous ions can be confined for a considerably long period, and as a mass spectrometer of large mass range variable mass resolution, and high sensitivity. As a storage device, the quadrupole ion trap confines gaseous ions, either positively or negatively charged and, when required, ions of each polarity

In 1956 Paul described a device containing gaseous ions in a small volume using only electric fields [32]. The quadrupole ion trap mass spectrometer consists essentially of three shaped electrodes 
that are shown in the dissembled structure in Figure 1-4. Two of the three electrodes are virtually identical and, while having hyperbolical geometry, these electrodes are referred to as endcap electrodes. The arrangement of the electrodes forms a chamber within which ions can be confined. Each endcap electrode consists of one or more holes in the center for injection or ejection of ions to or from the trap. One endcap electrode contains the "entrance" aperture in which gaseous phase ions can be gated periodically. The other endcap electrodes have "exit" apertures that facilitate ions passed to a detector. The ring electrode has an internal hyperbolical surface which is positioned symmetrically between the two endcap electrodes. Application of an r.f. voltage to the ring electrode establishes a quadrupole electric field (ideal situation) in which the force acting on an ion is inversely proportional to its square root of the distance from the center of the device; this allows ions of appropriate mass-to-charge ratios to have stable trajectories within the chamber and to be trapped for many seconds. For their pioneering work with ion traps, Wolfgang Paul of the University of Bonn and Hans Dehmelt of the University of Washington shared in the 1989 Nobel Prize for physics.

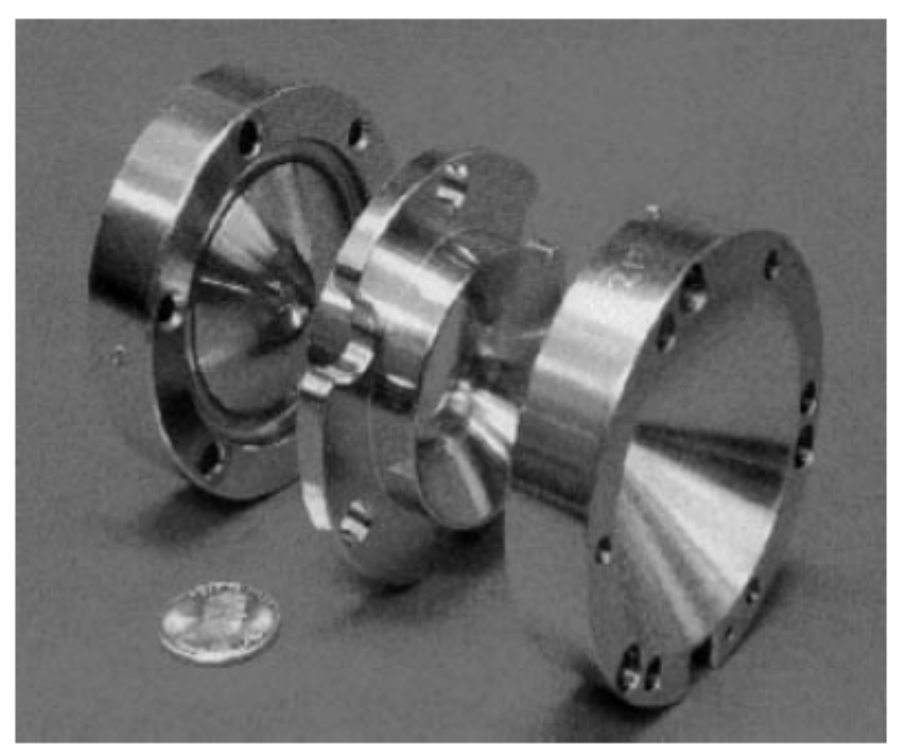


Figure 1-4: The three electrodes of the quadrupole ion trap shown in open array. (Reproduced from March and Todd (2005, Fig. 2.11 of QITMS), copyright at 2005, with permission of John Wiley \& Sons, Ltd.)

By application of an r.f. voltage of correct magnitude and frequency to the ring electrode, a broad range of ions with different $\mathrm{m} / \mathrm{z}$ can be trapped. For the detection of a mass spectrum, the amplitude of the r.f. voltage is increased with a predefined rate; this causes ions of increasing $\mathrm{m} / \mathrm{z}$ to become unstable as their excursions within the potential well created in the chamber increase in amplitude and eventually take them beyond the physical bounds of the device. At this point, they are ejected in mass sequence from the device through perforations in an endcap.

The first method of mass analysis done in the firstly developed quadrupole ion trap was mass selective detection. In mass selective ejection mode, the stable ion motion is sensed by the endcap electrodes. The main limitation of this technique was mass range and resolution which were limited as ions keep trapped during the analysis. The Finnigan Corporation's first design of the QIT had the limitations of mass range and resolution. The mass range and resolution of the QIT were improved when they introduced helium gas at about 1 mtorr to the vacuum system to couple the QIT with gas chromatography (GC) [33]. However, they encountered drawbacks of slight mass shifts for particular ions of a sample. The experimentations help them to bring a solution in which spacing out the endcap electrodes a bit from the earlier position could resolve the mass shift problem [33].

\subsubsection{Cylindrical Ion trap}

The cylindrical ion trap originated from the 3D quadrupole ion trap in which hyperbolic endcap electrodes and ring electrode have been replaced with a simplified planar electrode and 
cylindrical ring electrodes. This simple geometry makes it convenient to machine at small scale with high accuracy and precision which makes CIT an attractive candidate choice for the mass analyzer.

Langmuir et al. obtained a patent for a CIT in 1962 [34]. Benilan and Audoin have also given a reasonably acceptable detailed description of the theory for CIT [35] and Bonner et al. further explained how the performance of CIT was different from the QIT in 1977 [36]. In 1998 Badman et al. introduced a miniaturized version of CIT with a trapping radius of $2.5 \mathrm{~mm}$ [37]. However, this approach didn't show better resolution, $\Delta m / z=1.4 \mathrm{Th}$, than a full-sized trap. In 1999 Kornienko et al. came up with another miniaturized CIT with a trapping radius of $0.5 \mathrm{~mm}$ with improved resolution [38]. However, from an analytical perspective, CIT dimensions on the submillimeter scale exhibit poor analytical performance due to the Coulombic repulsion of ions confined at the trapping center. The Coulombic repulsion of ions at the trapping center causes poor sensitivity, mass resolution, and mass accuracy. Also, the electrodes miniaturized scale CITs need lower r.f. voltage and r.f. frequency. As a result, the pseudopotential well becomes shallower and the ion cloud occupies a larger proportion of the trap [39]. A larger trapping volume is required to minimize ions' Coulombic repulsion to achieve reasonably acceptable analytical performance from a highly miniaturized ion trap. Simulation-based analysis indicates that the number of trapped ions scales as the 1.55-1.75 power of $r_{o}$ [40]. This translates into lower sensitivity per miniature CITs due to a decreased number of ions of the chemical of interest that can be analyzed. Mather et al. determined stability diagrams for several ionic species confined within three ion traps having cylindrical geometry [41]. Fulford et al. described the operation of a CIT as a reactor for the study of ion/molecule reactions and reported on the application of resonant ion ejection to the elucidation of ion/molecule reaction mechanisms [42]. Jardino and co-workers examined the effective potential of a CIT and the spatial symmetry and 
harmonicity of that potential [43]. They found that the potential is not perfectly spherically symmetrical as in the case of the hyperbolic QIT. Nevertheless, they concluded that greater ion densities can be achieved, and a greater total number of ions confined in a CIT than in one possessing quadrupole geometry, as had been reported earlier [36].
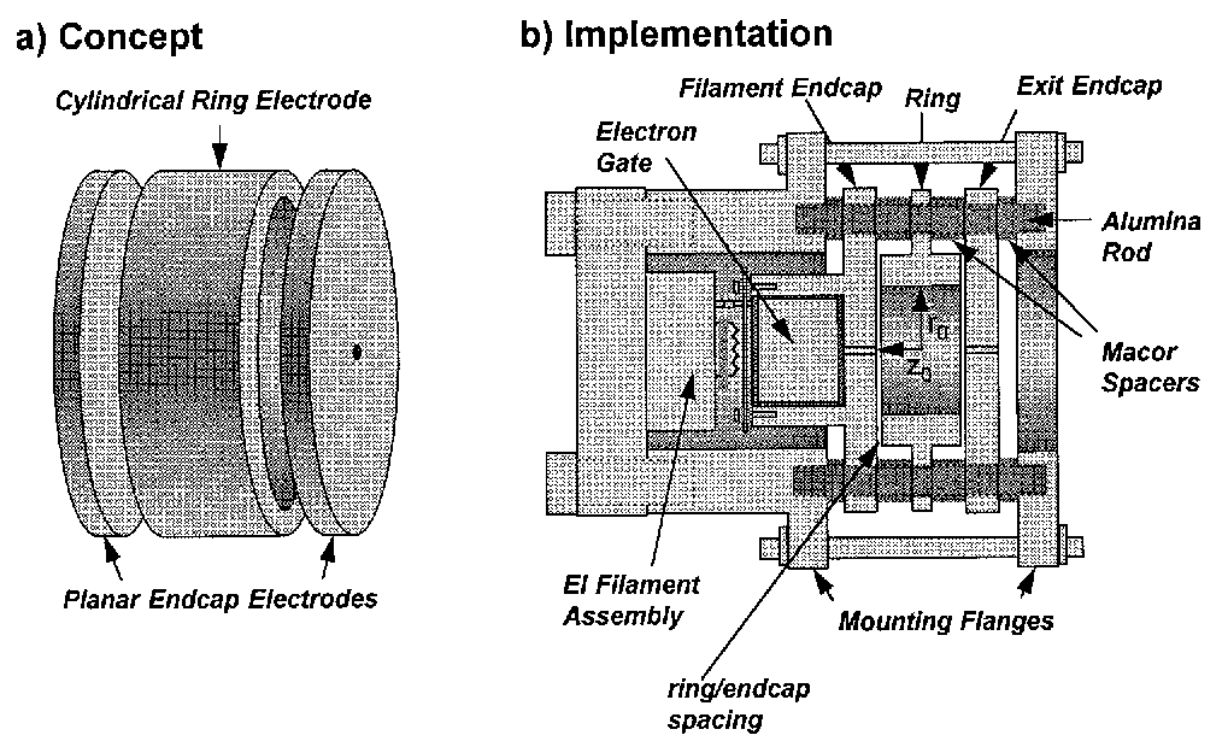

Figure 1-5 (a) Concept and (b) experimental implementation of a cylindrical ion trap as an approximation to the hyperbolic Paul ion trap. (Adapted from Wells, J. M.; Badman, E. R.; Cooks, R. G. A Quadrupole Ion Trap with Cylindrical Geometry Operated in the Mass-Selective Instability Mode. Anal. Chem. 1998, 70 (3), 438-444.)

\subsubsection{Linear Ion trap}

A Linear Ion Trap (LIT) with hyperbolic electrodes can be considered another ion trap design that could overcome the ion capacity seen in the QIT and CIT. The extended length in LIT can be utilized to increase the trapping volume; thereby, increased trapping volume spread more ions along the whole length at a given space charge effect. 


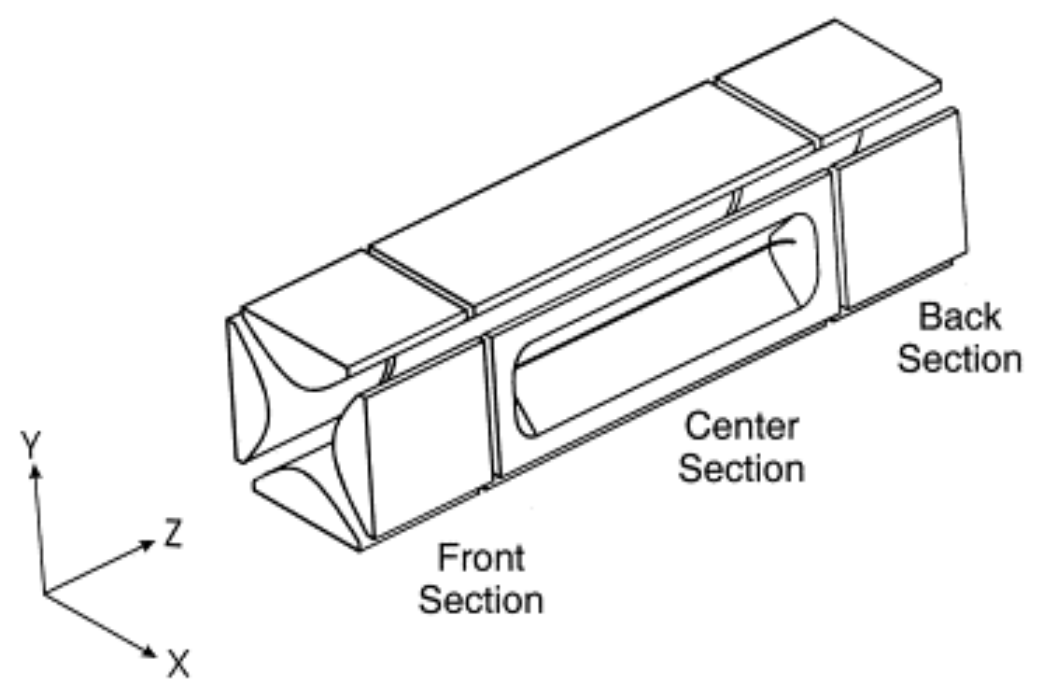

Figure 1-6: Basic design of the two-dimensional linear ion trap. (Adapted from Schwartz, J. C.; Senko, M. W.; Syka, J. E. P. A Two-Dimensional Quadrupole Ion Trap Mass Spectrometer. J. Am. Soc. Mass Spectrom. 2002, 13 (6), 659-669.)

The first linear traps were constructed in 1969 by Church in which bent linear quadrupoles into closed circle and "racetrack" geometries and demonstrated storage of ${ }^{3} \mathrm{He}^{+}$and $\mathrm{H}^{+}$ions for several minutes [44]. In 1989, Prestage, Dick, and Malecki described that ions could be trapped in the linear quadrupole trap system to enhance ion-molecule reactions; thus, it can be used to study spectroscopy of stored ions [45]. The linear ion trap consists of 2 sets of hyperbolic electrode rods to confine ion radially and axially with static and dynamic potential applied on each electrode. The field inside the trap creates a 2D electric field which allows ion motion in radial and axial directions. The ions can spread out the trapping region's whole length, allowing the traps to have high storage capacity [3]. The other main benefits of the LIT include the ease of coupling to other mass analyzers and higher trapping efficiency of externally generated ions and over $97 \%$ of ion ejection efficiency towards the detector. Also, the slits in axial and radial directions allow ion to be ejected in both directions. 
Two research groups trapped the ions in the collision cell of a tandem quadrupole mass filter to study and enhance the ion-molecule reactions. Since ions are spread out the whole length of trapping dimensions, the ions eject to a plane rather than to a point. In 1994, Bier and Syka patented this design for the Thermo Finnigan (Thermo Scientific) LIT [46]. In this arrangement of QMF sections, either of the last two sections can be operated as an ion trap, and an auxiliary AC applied can excite the ion motion of the matching secular frequency causing the ions to be ejected axially. However, electrode manufacturing in LIT can also be challenging in the submillimeter scale.

\subsubsection{Rectilinear Ion Trap}

The main drawback of all three-dimensional (3D) ion traps, including the CIT, is low trapping capacity and low trapping efficiency for externally injected ions [47]. The space charge effects result in diminished the mass resolution. The ion trapping efficiency for externally generated ions is also in the low range, 5\% [47]. To address such limitation in the quadrupole ion trap and cylindrical ion traps, Cooks' lab in 2004 was able to introduce another type of ion trap design [47]. This trap combines the features of the extended trapping capacity of LIT and the CIT's simplified electrode geometry. 


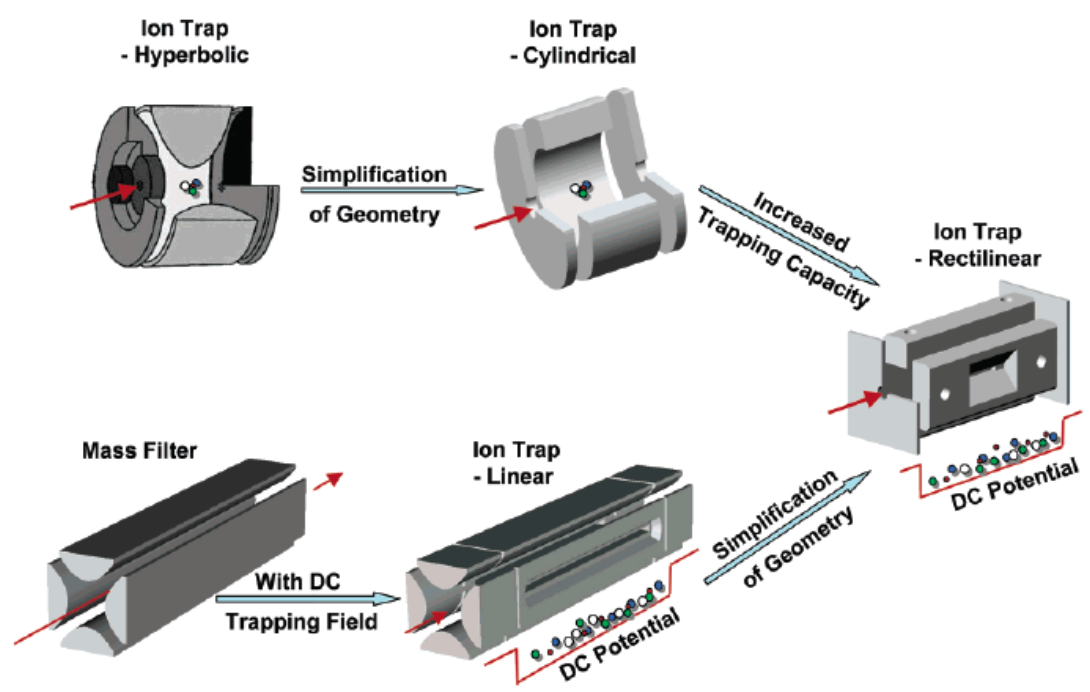

Figure 1-7: Conceptual evolution of the rectilinear ion trap and interrelationship to other types of ion traps (Adapted from Snyder, D. T.; Pulliam, C. J.; Ouyang, Z.; Cooks, R. G. Miniature and Fieldable Mass Spectrometers: Recent Advances. Anal. Chem. 2016, 88 (1), 2-29.)

In this approach, the hyperbolic electrodes' geometry has been replaced with simplified (planer) electrode geometry and two endcap electrodes are replaced with the help of planar electrodes including slits on each side to ions to come into the trap and eject the trap. In this geometry, the rectilinear ion trap consists of four $\mathrm{x}-\mathrm{y}$ facing planar electrodes that facilitate $2 \mathrm{D}$ trapping potential. Two endcap electrodes with simplified designing along the z-direction facilitate DC voltage to the system. The extended trapping dimensions along the z-direction is utilized to spread out the ions making trapping capacity 40 times greater than that of the CIT with the same size scale [47]. 


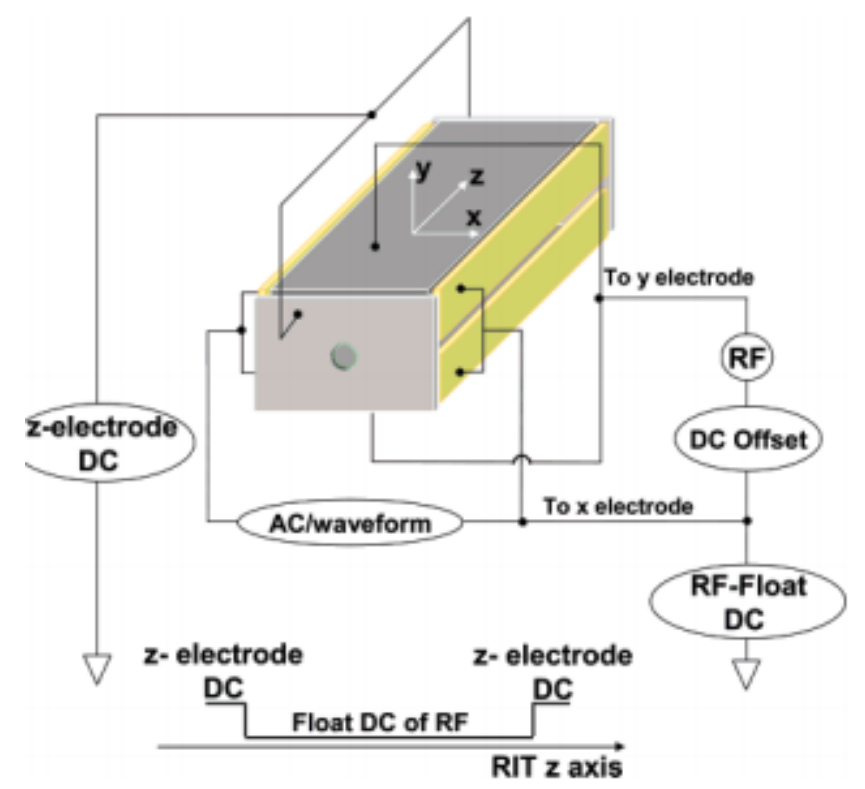

Figure 1-8: Configuration of the rectilinear ion trap and the control signals applied to it. (Ouyang, Z.; Wu, G.; Song, Y.; Li, H.; Plass, W. R.; Cooks, R. G. Rectilinear Ion Trap: Concepts, Calculations, and Analytical Performance of a New Mass Analyzer. Anal. Chem. 2004, 76 (16), 4595-4605.)

\subsubsection{Toroidal Ion trap}

In 2001, Lammert et al. develop and improved the first ion trap mass analyzer with a toroidal trapping volume [48]. The conceptual idea of Toroidal Ion Trap (TorIT) was initiated by certain other approaches including the patent of Bier and Syka [46], the effort of Dress and Paul [49], and Church's approach [44] to name a few. The patent of Bier and Syka reported several versions of linear quadrupole ion traps including one with bent electrodes [46]. This configuration is more efficient in ejecting ions into a point. The conventional LITs eject the ions in a plane in the radial direction. Such LITs have certain challenges, including larger detectors and low signal/noise ratio (SNR) due to the high dark current from the larger detector. All other approaches mentioned before are derived from the linear quadrupole mass filter by rearranging the electrodes into closed circle and 
"racetrack" configurations. By considering the Lammert's approach, the geometry of TorlT can be explained as a form of the derivation of the QIT in which the axial rotation of QIT was moved to a line outside of the cross-section of QIT. It can also be considered as a version of LIT in which electrodes' ends are interconnected. The formed geometry creates a cross-sectional shape like a torus for ions to occupy and spread along the trapping volume.

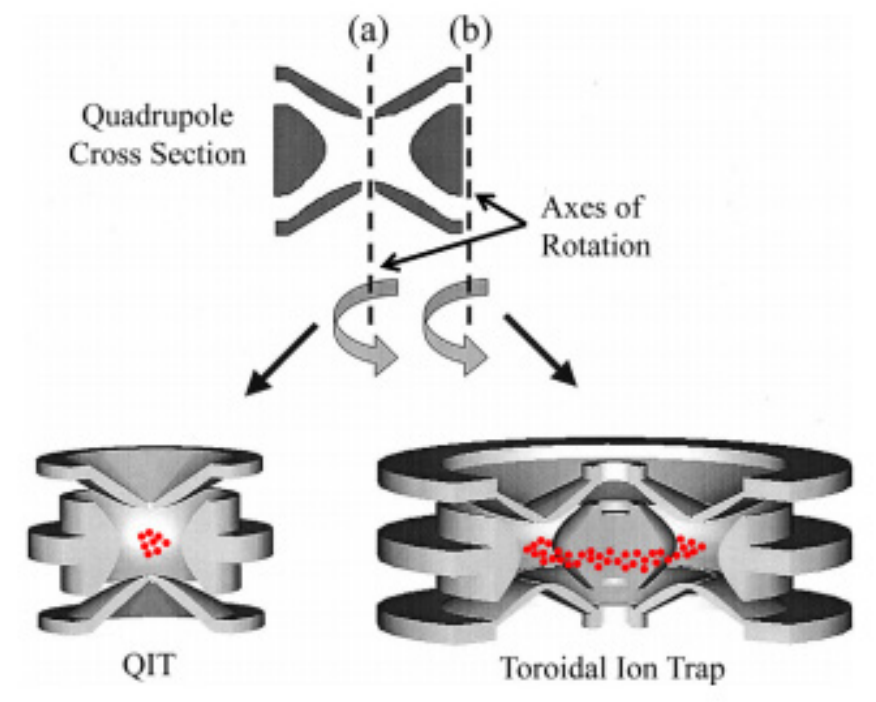

Figure 1-9: Comparison of the QIT and the toroidal ion trap. For the QIT, the rotational axis passes through the trapping center. (Adapted from Lammert, S. A.; Plass, W. R.; Thompson, C. V.; Wise, M. B. Design, Optimization and Initial Performance of a Toroidal r.f. Ion Trap Mass Spectrometer. Int. J. Mass Spectrom. 2001, 212 (1-3), 25-40.)

Many research groups have already taken the attention of TorIT due to the exciting features it offers such as improved trapping capacity, and improved SNR. These features make favorable conditions for the miniaturization of TorIT when optimized. The extended trapping volume can be generated by rotating the quadrupole cross-section at the edge axis or beyond the edge axis. This allows TorIT to have a larger ion trapping capacity by dispersing ions along the extended trapping volume compared to the other ion traps version with the same space charge effect. For example, a 
TorIT with a $10 \mathrm{~mm}$ trap radius, and $25.4 \mathrm{~mm}$ toroidal radius, can offer 240 times improved trapping capacity compared to the QIT with the same trap radius [48]. The space charging effect can also determine the limit of the dimensions of the ion trap mass analyzers. The relatively larger ion trapping capacity at a given space charge effect in TorlT allows us to build it at reduced dimensions. That leads to operating the TorIT at lower power, higher pressure and relatively lower voltage make favorable conditions to build a field-portable instrument. The other advantage of the TorIT with radial ejection is the requirement of a small detector. The small detector will reduce dark current which improves the SNR.

Because of the reasons mentioned above, TorIT has become one of the most popular radio frequency mass analyzers among certain developers and scientists. Various studies have been conducted to understand the physics behind the TorIT, simplification, and performance optimization of the TorIT mass analyzer. For example, the Lammert group customized the hyperbolic shape electrodes to rearrange the electric field to optimize the performance of the TorIT [48]. In addition to that, in 2009, Taylor and Austin presented a simplified version of the TorIT using only cylindrical and planar electrodes which are more convenient to fabricate than hyperbolic electrodes [50]. Another significant difference in Taylor's approach was the radial ejection in his design while Lammert used axial ejection. However, Taylor improved the simplified TorIT by optimizing the r.f. electrodes' spacing and setting a known amount of percentage of hexapole and octapole. In addition to these efforts, many other various studies have been done. For example, in 2010, Madsen and Gorman optimized the design parameters using finite-element analysis simulations to minimize higher-order anharmonic terms in the trapping pseudopotential [51]. Again, in Austin Lab, in 2013 and 2017, 
Higgs was able to first realize the motion of the ions in TorIT using ion trajectory simulation [52, $53]$.

Kotana and Mohanty introduced a mathematical approach to determine the multipole coefficients in the toroidal coordinate system [54]. Using their mathematical approach, they presented equations for ion motion, Mathieu parameters, and ions' secular frequency. In addition to these mentioned studies, many other optimizations and simplification studies can be found. As a result of all of these efforts, the TorIT mass analyzers are commercially available. For example, PerkinElmer was able to commercially introduce a novel portable gas chromatograph-mass spectrometer (Torion T-9 Portable GC/MS) based on a miniaturized, asymmetric TorIT mass analyzer.

\subsection{Ion Trajectory Simulation}

Simulation modeling can solve problems in the real world safely and efficiently at a low cost. It provides an important method of analysis that is easily verified, communicated, and understood. Across many wide varieties of industries and disciplines, simulation modeling can provide more detailed solutions by giving clear insights into complex systems that real modern technology cannot access easily. Most importantly, virtual experiments with simulation programs are less expensive and take less time than real experiments. Ion trajectory simulation is an essential and useful tool in instrumentation development for mass spectrometry. The ion trajectory simulation program in the mass spectrometry field can guide in understanding fundamental aspects of ion trap mass spectrometers. 


\subsubsection{History of SIMION}

SIMION is an ion optics simulation program that can be modeled ion optics problems with 2D symmetric and/or 3D asymmetric electrostatic and/or magnetic potential arrays. SIMION 3D is the most commonly used ion-optics simulation program in physics, chemistry, and engineering.

Don C. McGilvery, as a Ph.D. student under Professor James Morrison at La Trobe University, Melbourne, Australia developed the first version of SIMION in the late 1970s [55]. James Morrison and David A. Dahl later adapted SIMION for personal computers in 1985 at the Idaho National Engineering and Environmental laboratory [56]. Further, Richard Morrison and McGilvery developed a Macintosh version of SIMION known as MacSIMION at Monash University [57]. McGilvery and Dahl shared the distinguished contribution award from the American Society for Mass Spectrometry in 1998 to recognize the importance of their work [56].

\subsubsection{Working with SIMION}

The geometries of the electrodes can be made by entering them manually or with the help of a GEM file into a potential array (.PA or .PA\#). Before we draw the electrodes first the dimension of the potential array has to be defined in grid units (gu). Depending on the geometry of the electrodes, user may need to select planar or cylindrical symmetry. In SIMION, electrode shapes can be custom made by entering them into a potential array. Depending on the number of electrodes in the geometry, the user may need to assign different voltages for different electrodes. That can be facilitated by saving the potential array.PA\# format. The .PA\# file extension tells SIMION that this is a fast adjust 
definition file. Once the array is created, SIMION can refine the array by solving the Laplace equation by finite difference methods.

Once the refining process is done, now the user is allowed to scale the potential array from gu to mm. The "Particle" tab also allows the user to feed the information about the particle and initial operating condition that the user needs to run the simulation. Also, it facilitates the user to record the various type of parameters in different instances. Also, when ions fly at various times, SIMION may need to execute code in the user program attached to the current workbench. This program can monitor and change the state of the system. This enables users to customize and extend SIMION's calculation method in a very flexible way that hasn't been incorporated in SIMION default settings. For instance, the user can assign dynamic voltages for electrodes, introduce a collisional model with the background gas and data recording, and see a parameter's behavior as the effect of time-variant parameter changes. 


\subsection{References}

(1) Raymond, E. M.; John, F. T. Quadrupole Ion Trap Mass Spectrometry. In Chemical Analysis A series of monographs on analytical chemistry and its applications.; Winefordner, J. D., Ed.; John Wiley \& Sons: Hoboken, New Jersey, 2005.

(2) McLafferty, F. W. A Century of Progress in Molecular Mass Spectrometry. Annu. Rev. Anal. Chem. (Palo Alto Calif.) 2011, 4 (1), 1-22.

(3) Gao, L.; Song, Q.; Patterson, G. E.; Cooks, R. G.; Ouyang, Z. Handheld Rectilinear Ion Trap Mass Spectrometer. Anal. Chem. 2006, 78 (17), 5994-6002.

(4) Gao, L.; Sugiarto, A.; Harper, J. D.; Cooks, R. G.; Ouyang, Z. Design and Characterization of a Multisource Hand-Held Tandem Mass Spectrometer. Anal. Chem. 2008, 80 (19), 71987205.

(5) Li, L.; Chen, T.-C.; Ren, Y.; Hendricks, P. I.; Cooks, R. G.; Ouyang, Z. Mini 12, Miniature Mass Spectrometer for Clinical and Other Applications--Introduction and Characterization. Anal. Chem. 2014, 86 (6), 2909-2916.

(6) Contreras, J. A.; Murray, J. A.; Tolley, S. E.; Oliphant, J. L.; Tolley, H. D.; Lammert, S. A.; Lee, E. D.; Later, D. W.; Lee, M. L. Hand-Portable Gas Chromatograph-Toroidal Ion Trap Mass Spectrometer (GC-TMS) for Detection of Hazardous Compounds. J. Am. Soc. Mass Spectrom. 2008, 19 (10), 1425-1434.

(7) Shortt, B. J.; Darrach, M. R.; Holland, P. M.; Chutjian, A. Miniaturized System of a Gas Chromatograph Coupled with a Paul Ion Trap Mass Spectrometer. Journal of Mass Spectrometry 2005; 40: 36-42. 
(8) Chen, C.-H.; Chen, T.-C.; Zhou, X.; Kline-Schoder, R.; Sorensen, P.; Cooks, R. G.; Ouyang, Z. Design of Portable Mass Spectrometers with Handheld Probes: Aspects of the Sampling and Miniature Pumping Systems. J. Am. Soc. Mass Spectrom. 2015, 26 (2), 240-247.

(9) Paul, W.; Steinwedel, H. Notizen: Ein Neues Massenspektrometer Ohne Magnetfeld. Z. Naturforsch. A 1953, 8 (7).

(10) March, R. E.; Todd, J. F. Quadrupole Ion Trap Mass Spectrometry, 2nd ed.; WileyInterscience: New York, 2008.

(11) Stafford, G., Jr. Ion Trap Mass Spectrometry: A Personal Perspective. J. Am. Soc. Mass Spectrom. 2002, 13 (6), 589-596.

(12) Jonscher, K. R.; Yates, J. R., 3rd. The Quadrupole Ion Trap Mass Spectrometer--a Small Solution to a Big Challenge. Anal. Biochem. 1997, 244 (1), 1-15.

(13) Chattopadhyay, M.; Mohanty, A. K. Off-Axis Field Approximations for Ion Traps with Apertures. Int. J. Mass Spectrom. 2009, 288 (1-3), 58-67.

(14) Chattopadhyay, M.; Verma, N. K.; Mohanty, A. K. Composite Field Approximations for Ion Traps with Apertures on Electrodes. Int. J. Mass Spectrom. 2009, 282 (3), 112-122.

(15) Koizumi, H.; Whitten, W. B.; Reilly, P. T. A.; Koizumi, E. The Effect of Endcap Electrode Holes on the Resonant Ejection from an Ion Trap. Int. J. Mass Spectrom. 2009, 281 (3), 108114.

(16) Tallapragada, P. K.; Mohanty, A. K.; Chatterjee, A.; Menon, A. G. Geometry Optimization of Axially Symmetric Ion Traps. Int. J. Mass Spectrom. 2007, 264 (1), 38-52. 
(17) Wang, Y.; Zhang, X.; Feng, Y.; Shao, R.; Xiong, X.; Fang, X.; Deng, Y.; Xu, W. Characterization of Geometry Deviation Effects on Ion Trap Mass Analysis: A Comparison Study. Int. J. Mass Spectrom. 2014, 370, 125-131.

(18) Xu, W.; Chappell, W. J.; Cooks, R. G.; Ouyang, Z. Characterization of Electrode Surface Roughness and Its Impact on Ion Trap Mass Analysis. J. Mass Spectrom. 2009, 44 (3), 353360.

(19) Blain, M. G.; Riter, L. S.; Cruz, D.; Austin, D. E.; Wu, G.; Plass, W. R.; Cooks, R. G. Towards the Hand-Held Mass Spectrometer: Design Considerations, Simulation, and Fabrication of Micrometer-Scaled Cylindrical Ion Traps. Int. J. Mass Spectrom. 2004, $236(1-3), 91-104$.

(20) Chaudhary, A.; van Amerom, F. H. W.; Short, R. T.; Bhansali, S. Fabrication and Testing of a Miniature Cylindrical Ion Trap Mass Spectrometer Constructed from Low Temperature Co-Fired Ceramics. Int. J. Mass Spectrom. 2006, 251 (1), 32-39.

(21) Gamage, R. W.; Austin, D. E. The Effects of Electrode Misalignments on the Performance of a Miniaturized Linear Wire Ion Trap Mass Spectrometer. Int. J. Mass Spectrom. 2020, 453 (116344), 116344.

(22) Konenkov, A. N.; Douglas, D. J.; Konenkov, N. V. Spatial Harmonics of Linear Multipoles with Round Electrodes. Int. J. Mass Spectrom. 2010, 289 (2-3), 144-149.

(23) Wu, G.; Cooks, R. G.; Ouyang, Z. Geometry Optimization for the Cylindrical Ion Trap: Field Calculations, Simulations and Experiments. Int. J. Mass Spectrom. 2005, 241 (2-3), 119132. 
(24) Franzen, J. The Non-Linear Ion Trap. Part 4. Mass Selective Instability Scan with Multipole Superposition. Int. J. Mass Spectrom. Ion Process. 1993, 125 (2-3), 165-170.

(25) Franzen, J. The Non-Linear Ion Trap. Part 5. Nature of Non-Linear Resonances and Resonant Ion Ejection. Int. J. Mass Spectrom. Ion Process. 1994, 130 (1-2), 15-40.

(26) Franzen, J. Simulation Study of an Ion Cage with Superimposed Multipole Fields. Int. J. Mass Spectrom. Ion Process. 1991, 106, 63-78

(27) Guzowski, J. P., Jr; Hieftje, G. M. Characteristics of a Rf-Only Hexapole Ion-Guide Interface for Plasma-Source Time-of-Flight Mass Spectrometry. J. Anal. At. Spectrom. 2001, 16 (8), $781-792$.

(28) Tosi, P.; Fontana, G.; Longano, S.; Bassi, D. Transport of an Ion Beam through an Octopole Guide Operating in the R.F.-Only Mode. Int. J. Mass Spectrom. Ion Process. 1989, 93 (1), $95-105$.

(29) Hägg, C.; Szabo, I. New Ion-Optical Devices Utilizing Oscillatory Electric Fields. II. Stability of Ion Motion in a Two-Dimensional Hexapole Field. Int. J. Mass Spectrom. Ion Process. 1986, 73 (3), 237-275.

(30) Hägg, C.; Szabo, I. New Ion-Optical Devices Utilizing Oscillatory Electric Fields. III. Stability of Ion Motion in a Two-Dimensional Octopole Field. Int. J. Mass Spectrom. Ion Process. 1986, 73 (3), 277-294.

(31) Practical Aspects of Ion Trap Mass Spectrometry, Volume I; March, R. E., Todd, J. F. J., Eds.; CRC Press: Boca Raton, FL, 1995.

(32) Snyder, D. T.; Cooks, R. G. Successive Resonances for Ion Ejection at Arbitrary Frequencies in an Ion Trap. J. Am. Soc. Mass Spectrom. 2016, 27 (12), 1922-1928. 
(33) Wolfgang, P.; Helmut, S. Apparatus for Separating Charged Particles of Different Specific Charges. U.S. Patent 2,939,952A, July 06, 1960.

(34) Plass, W. R.; Li, H.; Cooks, R. G. Theory, Simulation and Measurement of Chemical Mass Shifts in RF Quadrupole Ion Traps. Int. J. Mass Spectrom. 2003, 228 (2-3), 237-267.

(35) Shindler, H. A. Containment Area Device. U.S. Patent 3,148,699A, September 15, 1964.

(36) Benilan, M.-N.; Audoin, C. Confinement d'ions par un champ électrique de radio-fréquence dans une cage cylindrique. Int. j. Mass spectrom. Ion Phys. 1973, 11 (5), 421-432.

(37) Bonner, R. F.; Fulford, J. E.; March, R. E.; Hamilton, G. F. The Cylindrical Ion Trap. Part I. General Introduction. Int.j. mass spectrom. ion phys. 1977, 24 (3), 255-269.

(38) Badman, E. R.; Johnson, R. C.; Plass, W. R.; Cooks, R. G. A Miniature Cylindrical Quadrupole Ion Trap: Simulation and Experiment. Anal. Chem. 1998, 70 (23), 4896-4901.

(39) Kornienko, O.; Reilly, P. T. A.; Whitten, W. B.; Ramsey, J. M. Micro Ion Trap Mass Spectrometry. Rapid Commun. Mass Spectrom. 2004, 18 (6), 742-742.

(40) Tian, Y.; Higgs, J.; Li, A.; Barney, B.; Austin, D. E. How Far Can Ion Trap Miniaturization Go? Parameter Scaling and Space-Charge Limits for Very Small Cylindrical Ion Traps: Ion Trap Miniaturization. J. Mass Spectrom. 2014, 49 (3), 233-240.

(41) Chernookiy, D. Optimization of the Cylindrical Ion Trap Geometry For Mass Analysis at High Pressure, University of North Carolina, Chapel Hill, 2016.

(42) Mather, R. E.; Waldren, R. M.; Todd, J. F. J.; March, R. E. Some Operational Characteristics of a Quadrupole Ion Storage Mass Spectrometer Having Cylindrical Geometry. Int. j. Mass Spectrom. Ion Phys. 1980, 33 (3), 201-230. 
(43) Gabling, R.-H.; Romanowski, G.; Wanczek, K.-P. A Tandem Cluster-Beam-QuistorQuadrupole Instrument for the Study of Cluster Ions. Int. J. Mass Spectrom. Ion Process. 1986, 69 (2), 153-162.

(44) Lagadec, H.; Meis, C.; Jardino, M. Effective Potential of an Rf Cylindrical Trap. Int. J. Mass Spectrom. Ion Process. 1988, 85 (3), 287-299.

(45) Church, D. A. Storage-ring Ion Trap Derived from the Linear Quadrupole Radio-frequency Mass Filter. J. Appl. Phys. 1969, 40 (8), 3127-3134.

(46) Prestage, J. D.; Dick, G. J.; Maleki, L. New Ion Trap for Frequency Standard Applications. J. Appl. Phys. 1989, 66 (3), 1013-1017.

(47) Mark E. Bier John E. P. Syka. Ion Trap Mass Spectrometer System and Method. 5420425A, 1995.

(48) Ouyang, Z.; Wu, G.; Song, Y.; Li, H.; Plass, W. R.; Cooks, R. G. Rectilinear Ion Trap: Concepts, Calculations, and Analytical Performance of a New Mass Analyzer. Anal. Chem. 2004, 76 (16), 4595-4605.

(49) Lammert, S. A.; Plass, W. R.; Thompson, C. V.; Wise, M. B. Design, Optimization and Initial Performance of a Toroidal Rf Ion Trap Mass Spectrometer. Int. J. Mass Spectrom. 2001, 212 (1-3), 25-40.

(50) Drees, J.; Paul, W. Beschleunigung von Elektronen in einem Plasmabetatron. Eur. Phys. J. A 1964, 180 (4), 340-361.

(51) Taylor, N.; Austin, D. E. A Simplified Toroidal Ion Trap Mass Analyzer. Int. J. Mass Spectrom. 2012, 321-322, 25-32. 
(52) Madsen, M. J.; Gorman, C. H. Compact Toroidal Ion-Trap Design and Optimization. Phys. Rev. A 2010, 82,043423

(53) Li, A.; Higgs, J. M.; Austin, D. E. Chaotic Motion of Single Ions in a Toroidal Ion Trap Mass Analyzer. Int. J. Mass Spectrom. 2017, 421, 95-103.

(54) Higgs, J. M.; Austin, D. E. Simulations of Ion Motion in Toroidal Ion Traps. Int. J. Mass Spectrom. 2014, 363, 40-51.

(55) Kotana, A. N.; Mohanty, A. K. Determination of Multipole Coefficients in Toroidal Ion Trap Mass Analysers. Int. J. Mass Spectrom. 2016, 408, 62-76.

(56) Enke, C. G.; Yost, R. A. Jim Morrison, Friend and Colleague. J. Am. Soc. Mass Spectrom. 2013, 24 (9), 1319-1323.

(57) Dahl, D. A. Simion for the Personal Computer in Reflection. Int. J. Mass Spectrom. 2000, 200 (1-3), 3-25.

(58) Downard, K. M.; de Laeter, J. R. A History of Mass Spectrometry in Australia. J. Mass Spectrom. 2005, 40 (9), 1123-1139.

(59) Austin, D. E.; Wang, M.; Tolley, S. E.; Maas, J. D.; Hawkins, A. R.; Rockwood, A. L.; Tolley, H. D.; Lee, E. D.; Lee, M. L. Halo Ion Trap Mass Spectrometer. Anal. Chem. 2007, 79 (7), 2927-2932.

(60) Badman, E. R.; Cooks, R. G. A Parallel Miniature Cylindrical Ion Trap Array. Anal. Chem. 2000, 72 (14), 3291-3297.

(61) Lammert, S. A.; Rockwood, A. A.; Wang, M.; Lee, M. L.; Lee, E. D.; Tolley, S. E.; Oliphant, J. R.; Jones, J. L.; Waite, R. W. Miniature Toroidal Radio Frequency Ion Trap Mass Analyzer. J. Am. Soc. Mass Spectrom. 2006, 17 (7), 916-922. 
(62) Patterson, G. E.; Guymon, A. J.; Riter, L. S.; Everly, M.; Griep-Raming, J.; Laughlin, B. C.; Ouyang, Z.; Cooks, R. G. Miniature Cylindrical Ion Trap Mass Spectrometer. Anal. Chem. 2002, 74 (24), 6145-6153.

(63) Wu, Q.; Li, A.; Tian, Y.; Zare, R. N.; Austin, D. E. Miniaturized Linear Wire Ion Trap Mass Analyzer. Anal. Chem. 2016, 88 (15), 7800-7806.

(64) Peng, Y.; Hansen, B. J.; Quist, H.; Zhang, Z.; Wang, M.; Hawkins, A. R.; Austin, D. E. Coaxial Ion Trap Mass Spectrometer: Concentric Toroidal and Quadrupolar Trapping Regions. Anal. Chem. 2011, 83 (14), 5578-5584.

(65) Beaty, E. C. Calculated Electrostatic Properties of Ion Traps. Phys. Rev. A Gen. Phys. 1986, 33 (6), 3645-3656. 
2 VARYING THE ASPECT RATIO OF TOROIDAL ION TRAPS: IMPLICATIONS FOR DESIGN, PERFORMANCE, AND MINIATURIZATION

(This chapter has been prepared for submission as an article: "Hettikankanange, P.M., Austin, D. E. Varying the aspect ratio of toroidal ion traps: implications for design, performance, and miniaturization." My contribution was creating the electrode shapes for simulation using .gem file, simulating ion motion, apply the hard-sphere collision model, generate the frequency spectrum, find the secular frequency, construct the polynomial voltage profile, and analyze data and writing the draft manuscript.) 


\subsection{Introduction}

Portable mass spectrometry seeks to make real-time, in situ chemical measurements of complex or rapidly varying systems in their original environment, promising significant opportunities and advantages in many fields of science. Many advances in the development of portable mass spectrometers have been driven by the challenges of maintaining analytical performance while making instruments smaller, lighter, and more rugged, while others are based on reducing the cost and operational complexity of such systems to promote wider use [1-7]. Ion traps are one of the most common types of mass analyzers used in miniaturization efforts due to their inherently small size, tolerance to higher pressures, and ability to perform tandem mass analysis on complex samples. However, ion traps are by no means simple devices, and miniaturization efforts have led to many changes and improvements in trap design and operation. For example, simplifying the electrode geometry is of particular utility for miniaturizing ion trap mass analyzers $[5,6,8]$, because the ideal hyperbolic electrode shapes are difficult to produce accurately at small scales. Cylindrical ion traps (CITs) and the rectilinear ion trap (RIT) address this particular issue, as the planar and cylindrical electrode shapes can be made with better precision. Electrode positioning and alignment, and even surface smoothness can all affect the ion trap performance and become more significant at smaller scales $[9,10]$.

As an example of another issue, reducing the physical size of the ion trap allows for lower RF voltages and higher-pressure operation, but comes at the cost of reduced trapping capacity and reduced sensitivity. Parallel arrays of cylindrical ion traps increase total trapping capacity by having multiple traps, although any dimensional variation between traps results in reduced mass resolution [2]. The issue of reduced trapping capacity is also addressed in ion traps with an extended trapping dimension, such as toroidal $[1,4,11,12]$, linear, and rectilinear ion traps [8]. Trapped ions are able to spread out along this extended trapping dimension, reducing space-charge effects and allowing more ions to be trapped. At the same time, the trapping 
voltage and frequency--and typically the tolerable operating pressure--are governed by the characteristic trapping dimension, which is typically the dimension of ion ejection. The characteristic trapping dimension is perpendicular to the extended trapping dimension. For a miniaturized device, this characteristic trapping dimension is reduced, realizing the benefits of lower electrical power and higher operating pressure, while the trapping capacity is retained through the extended trapping dimension.

The aspect ratio (AR) of such an ion trap is defined as the ratio between the extended trapping dimension and the characteristic trapping dimension. Although a high aspect ratio would seem to offer the best of both worlds - the benefits of miniaturization without the loss of sensitivity—several practical issues must also be addressed. For example, the tolerable misalignment of electrodes scales with the characteristic trapping dimension, becoming more difficult for high aspect ratio traps [9-13]. Collecting ions ejected from a long ejection slit onto a small detector may also present ion focusing challenges. In the case of linear and rectilinear traps, axial trapping using DC potentials on endcaps concentrates ions at the trap center, but this increases space-charge effects and reduces trapping capacity. Toroidal ion traps are able to focus ions radially onto a small detector, but changing the aspect ratio also changes the shape of the trapping potential itself.

Toroidal ion traps (TorIT) are based on a quadrupole-like cross-section but rotated using an axis of rotation located outside of the quadrupole cross-section [12] (Figure 1). Thus, the aspect ratio of a TorIT can be defined as the ratio between the trap's annular radius (inscribed toroidal radius, $\left.R_{0}\right)$ to the characteristic trapping radius $\left(r_{0}\right)$. The extended trapping dimension has length $2 \pi R$, and ions are able to spread out along the entire ring. As with the other types of ion traps, a version of the toroidal ion trap has been developed using simplified geometry (the Simplified Toroidal Ion Trap, or STorIT), with all electrodes either as planar or cylindrical 
surfaces [6]. In the asymmetric TorIT, two different asymptote angles were assigned for the electrode geometry: one for surfaces located within $R_{0}$ and the second for surfaces beyond $R_{0}$ [12].

In contrast to all other types of ion traps, changing the aspect ratio of a toroidal ion trap results in changes to the degree of curvature and the shape of the trapping potential and, ultimately, the performance as a mass analyzer. Understanding these changes, including the higher-order terms in the trapping potential, are important to understand the relationship between performance and aspect ratio, and are key to developing an instrument that benefits from lower power and higher-pressure operation while maximizing sensitivity. Previous studies have shown that the curvature of the toroidal trapping region results in several effects. For example, Lammert showed that the symmetric TorIT suffers from poor mass resolution because of the additional fields contributed by the curvature of the device [12]. Higgs and Austin showed using ion trajectory simulations that the curvature of the trap causes ions to experience a centripetal-like effect which can shift the ion motion outward [14]. The effects of curvature will be directly impacted by the choice of aspect ratio, and will impact performance as a miniaturized device. Finally, as the AR of the toroidal trap becomes very large, as may be done to increase trapping capacity, curvature effects disappear and the fields within the device look more and more like those of a LIT. 
a

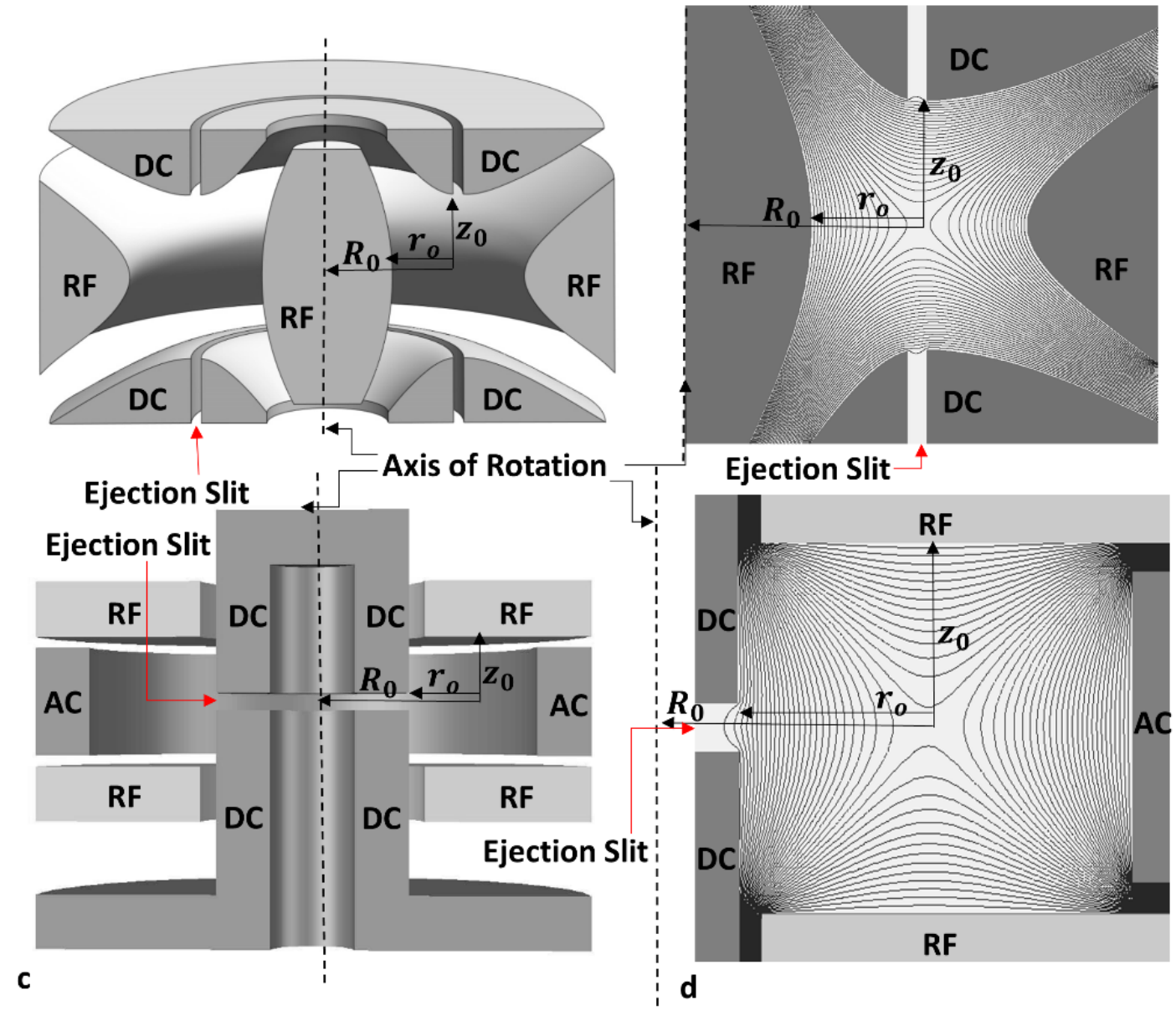

Figure 1: Cross-section views of asymmetric TorIT (a) and simplified TorIT (b) with DC and RF electrodes. The dimensions for the asymmetric TorIT and simplified TorIT were taken from Lammert et al. [12] and Taylor and Austin [6], respectively. The aspect ratio (AR) of any TorIT is defined as the ratio between the annular radius (inscribed toroidal radius) $\left(R_{0}\right)$ to the trap radius $\left(r_{0}\right)$. Images (c) and (d) represents the trapping regions with iso-potential contours of asymmetric and simplified TorIT respectively.

In this study, we analyze the response of key TorIT parameters to the change of AR. We also compare the response of these parameters with two different TorIT designs including the simplified TorIT with radial ejection and the asymmetric TorIT with axial ejection. We observe changes in the higher-order terms, shift of the saddle point compared to the geometric trapping center, and dependency of the secular frequency with respect to AR. Understanding such parameters with AR could help to optimize the performance of the trap in the design stage and 
provide a foundation to develop a mathematical relationship between the stability parameters and AR.

\subsection{Methods}

SIMION 8.0 (Scientific Instrument Services, Ringoes, NJ) was used to simulate the behavior and performance of the asymmetric and simplified TorIT with different aspect ratios.

The simplified TorIT dimensions used for the simulations in this project were taken from Taylor and Austin [6] while the asymmetric TorIT dimensions were taken from Lammert et al. [12]. The aspect ratios of these traps as originally presented were 2.54 (asymmetric) and 6.1 (simplified). However, we investigated the effect of ARs starting from 1.86 and increasing until 47.75, by which point the AR-dependence of all parameters became negligible. Consistent with the above references, we used radial ejection in the simplified TorIT and axial ejection in the asymmetric trap. Due to reflection symmetry about the radial plane, the saddle point of the trapping potential in the simplified TorIT always aligns exactly with the ejection slit. However, in the asymmetric TorIT with axial ejection, the position of the saddle point changes with the AR. For these simulations, we positioned the exit slits directly above and below the saddle point (more correctly, a saddle ring) in the potential. The location of the slits was adjusted for each AR studied to keep them aligned exactly with the trapping center.

The scale used both for the simplified and asymmetric TorIT simulations was 0.0125 $\mathrm{mm}$ per grid unit. The symmetry of the electrodes in SIMION was set to cylindrical. For evaluating the shape of the trapping potential distribution, the r.f. electrodes were set to $500 \mathrm{~V}$, with $0 \mathrm{~V}$ applied to the DC electrodes. The potential was recorded at each point along the ejection direction of the trap, following a linear trajectory from the center of the slit to the other 
end on the electrode through the geometric trapping center. The potential was recorded at each grid unit.

The resulting data was then imported into MATLAB R2017a (The Math Works, Natick, MD) for analysis. The positions in the ejection direction were normalized relative to the trap radius. The reason for the use of normalized positions along the ejection direction is to avoid the higher-order terms having different units. The potential profile along the ejection direction was analyzed by fitting the normalized points to a polynomial function. The built-in polyfit function in MATLAB was used to construct the polynomial function. Rather than using all data points contained within the trapping region, only the 400 points centered on the saddle point (out of 1000 points total) were used for the higher-order multipole calculation. The reason for using a subset of data points was to avoid the fringe effect near the electrodes. Many other efforts to evaluate higher-order multipoles have fitted data to a 25 th order polynomial function $[[6],[9], 14,15]$. To determine the optimal order of the polynomial function for a given set of data, the variance of the polynomial function was examined. The response of the variance was investigated from $6^{\text {th }}$ order to $25^{\text {th }}$ order with different numbers of data points which are close to and on both sides of the saddle point. According to this variance analysis, the 8th order polynomial fit with 400 data points (representing $40 \%$ of the entire data set) gave the lowest variance compared to other polynomial function orders and different fractions of the total data set. The coefficients obtained from the polynomial function were then used to calculate the magnitude of the hexapole $\left(\mathrm{A}_{6}\right)$ octopole $\left(\mathrm{A}_{8}\right)$, decapole $\left(\mathrm{A}_{10}\right)$, and dodecapole $\left(\mathrm{A}_{12}\right)$ components. Higher-order field contributions $\left(\mathrm{A}_{\mathrm{n}} / \mathrm{A}_{2} \%\right)$ were then calculated by taking the percentage relative to the quadrupole $\left(\mathrm{A}_{2}\right)$ term. We note that the conventional multipoles used to describe other types of ion traps are not mathematically valid for toroidal traps: at the axis of rotation, a non-zero electric field cannot be continuous and differentiable, violating the 
Laplace equation. Nonetheless, expressing the conventional multipoles in the vicinity of the trapping center is still useful to help qualitatively understand ion behavior in toroidal traps.

To investigate the secular frequency of ions with no collision gas, for each trap geometry an ion with $\mathrm{m} / \mathrm{z}=100 \mathrm{Th}$ was simulated with the trap operating at r.f. $=500 \mathrm{~V}_{0-\mathrm{p}}$ and a frequency of $1 \mathrm{MHz}$. The initial velocity components of each ion along the radial, axial and tangential directions were set to $0.1 \mathrm{~mm} / \mathrm{ms}$. In each simulations the ion was introduced to a point $0.5 \mathrm{~mm}$ away from the saddle point towards the outer electrode. The position $(\mathrm{x}, \mathrm{y}, \mathrm{z})$ of the ion was recorded every $0.01 \mu$ s until 8192 data points were recorded. Because $2^{\mathrm{n}}$ number of data points improves the accuracy of the Fast Fourier Transform function (FFT). The coordinates of the position of the ion with respect to time were converted to axial, angular, and radial positions, and the average radial position was also calculated. The frequency spectrum of the ion was then evaluated with MATLAB's $\boldsymbol{F F T}$ to identify the frequencies of the ion motion.

Ion ejection in the simplified TorIT was investigated using mass-selective ejection under the same conditions used above, with an auxiliary AC voltage applied on the outer electrode $\left(V_{0-p}=2 V\right)$ to excite the ion via dipolar resonant ejection. The frequency for the AC voltage was set to a value that is a few percent lower than the exact secular frequency of the ion [16]. This percentage was the minimum percentage that required eject the ion from the trap, which was manually determined by running multiple simulation runs. Collision effects were incorporated into the SIMION user program using a hard-sphere model. The pressure and temperature of the system were set to $1.5 \mathrm{mTorr}$ and $273 \mathrm{~K}$. The number of ions ejected from the trap were calculated by adding a part of user program to the main .LUA program. 


\subsection{Results and Discussion}

The polynomial equations from the potential mapping in both designs were used to determine the higher-order field contribution of $A_{3}, A_{4}, A_{5}$, and $A_{6}$ respect to the $A_{2}$ term. Figures $2 \mathrm{a}$ and $2 \mathrm{~b}$ show the dependency of higher-order terms with AR in the simplified TorIT with radial ejection and the asymmetric TorIT with axial ejection. The simplified TorIT with radial ejection has contributions from odd-order terms along the radial direction, as shown in Fig. 2(a). For ion traps with reflection symmetry about the central plane, the dipole term $\left(A_{1}\right)$, and all higher odd-order terms vanish due to symmetry [14]. This is the case for axial ejection in the asymmetric ion trap (Fig. 2b) where all the odd-order terms including hexapole and decapole terms are approximately zero regardless of the AR. In this case, there are still curvature effects [14], but they are not represented in the odd-order terms of the trapping potential because the multipole expansion is oriented in the axial rather than radial directions.

Non-linearity of the electric field in an ion trap is typically caused by ejection holes or slits, by electrode truncation [17], by irregularities on the electrode surface [10], by misalignment of the electrodes [9-13], or by non-ideal electrode shape or spacing. For toroidal traps, the curvature of the toroidal trapping region also affects field non-linearity $[12,14]$. At lower ARs, the degree of the curvature of the inner and outer electrodes are very significant. The curvature effects can strongly interact with the ions in the ion cloud. Also, the curvature effect is less significant at higher ARs. Further, we can hypothesize that the curvature effect has a less significant contribution to the quadrupole potential in TorITs when AR tends to infinity.

We can use the above explanation to describe the trend of the higher-order terms with ARs. According to Fig. 2a and Fig. 2b, the magnitudes of higher-order terms are significant 
due to the strong influence of the curvatures of inner and outer electrodes. The higher-order terms are flattened off at higher ARs as a result of a minor influence of the curvature effect.
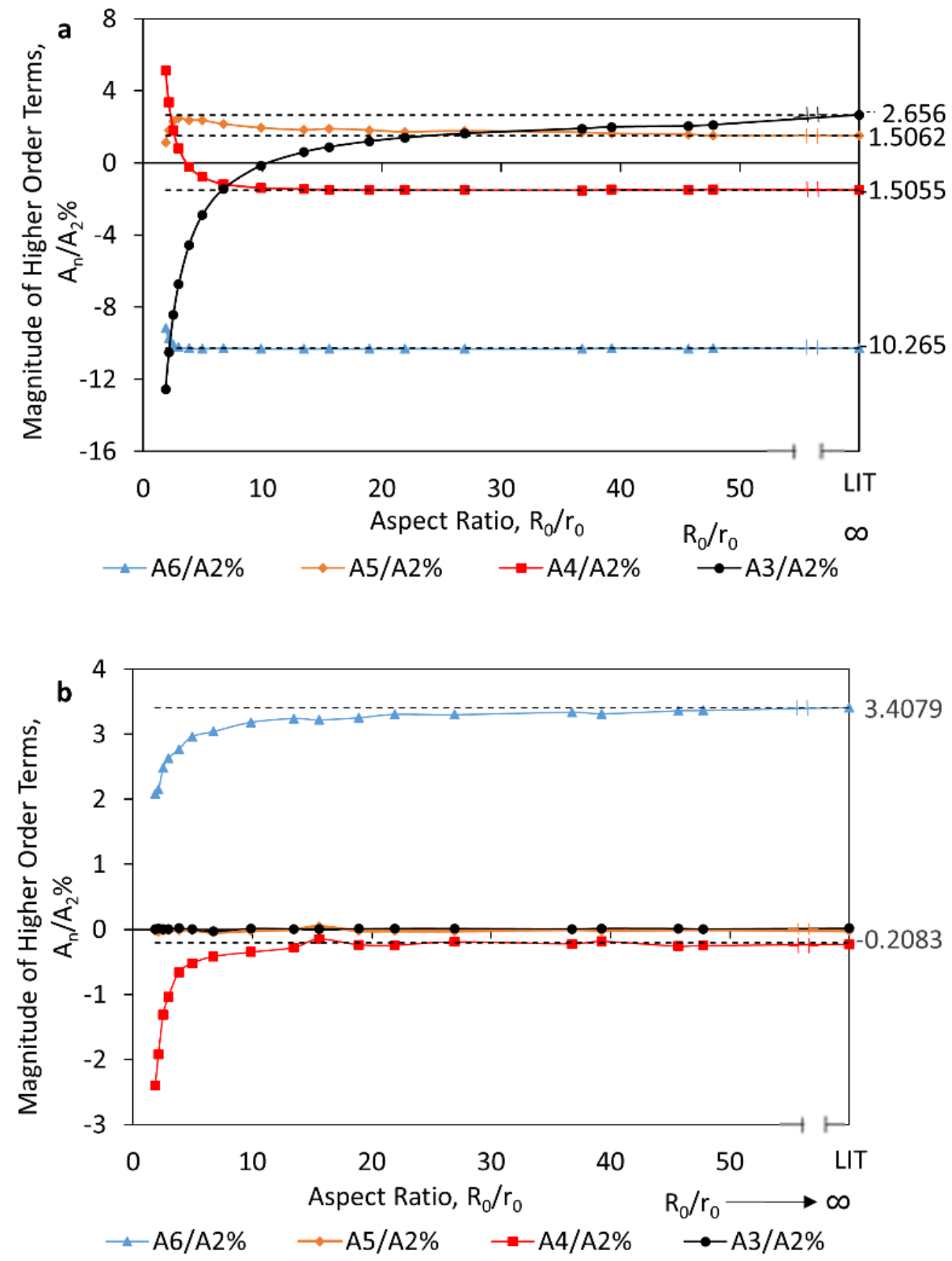

Figure 2: Effect of aspect ratio on the percent hexapole $\left[\left(\mathrm{A}_{3} / \mathrm{A}_{2}\right) \times 100 \%\right]$, octopole $\left[\left(\mathrm{A}_{4} / \mathrm{A}_{2}\right)\right.$ $\times 100 \%$, decapole $\left[\left(\mathrm{A}_{5} / \mathrm{A}_{2}\right) \times 100 \%\right]$, and dodecapole $\left[\left(\mathrm{A}_{6} / \mathrm{A}_{2}\right) \times 100 \%\right]$ in the simplified TorIT in radial direction (a) and asymmetric TorIT in axial direction (b). The trapping radius, $\mathrm{r}_{0}$, was held constant at $10 \mathrm{~mm}$ and $5.8 \mathrm{~mm}$ for asymmetric TorIT and simplified TorIT respectively. The last points represent the higher-order terms of a linear-type trap with the electrode crosssection of the corresponding toroidal trap. 
As discussed, the curvature effect can superimpose non-linear electric fields to the TorIT. Also, it can alter the location of the saddle point of a TorIT. The saddle point is the point where the potential reaches its maximum or minimum in the trapping region. Due to the symmetric nature, the saddle point and geometric trap center coincide in symmetric QIT and LIT. However, SIMION simulations of simplified TorIT with radial ejection system and asymmetric TorIT with axial ejection system show that there is no requirement to coincide the geometric trapping center with the saddle point. Kotana and Mohanty also provided that the geometric trap center and saddle point do not coincide [18]. So, we believe that the saddle point that is off from the geometric trap center also arises due to the curvature effect. Understanding of separation of geometric trap center and saddle point is a key phenomenon in the trap designing stage as it could determine the performance of the trap. Also, this understanding is very important in TorITs with ion ejection along the axial direction. The saddle point of simplified and asymmetric TorIT always in the $\mathrm{z}=0$ plane and stays at different positions along the $\mathrm{z}=0$ plane with AR as the degree of curvature changes. In practice, the ejection slits of an ion trap align with the saddle point. Lack of understanding of the location of the saddle point could result in wider exit slits. The wider slits act strongly on the trapping potential trap. Also, mass spectrometers with wider slits suffer in mass resolution. Properly aligned saddle point and exit slit could improve the sensitivity of the instrument by increasing the ion ejection efficiency. Therefore, understanding the separation of the saddle point from the geometric trapping center with $\mathrm{AR}$ is important in predicting the magnitude of higher-order terms, ion ejection efficiency, and mass resolution. Further, this information helps to optimize the ion trap in the design stage. 


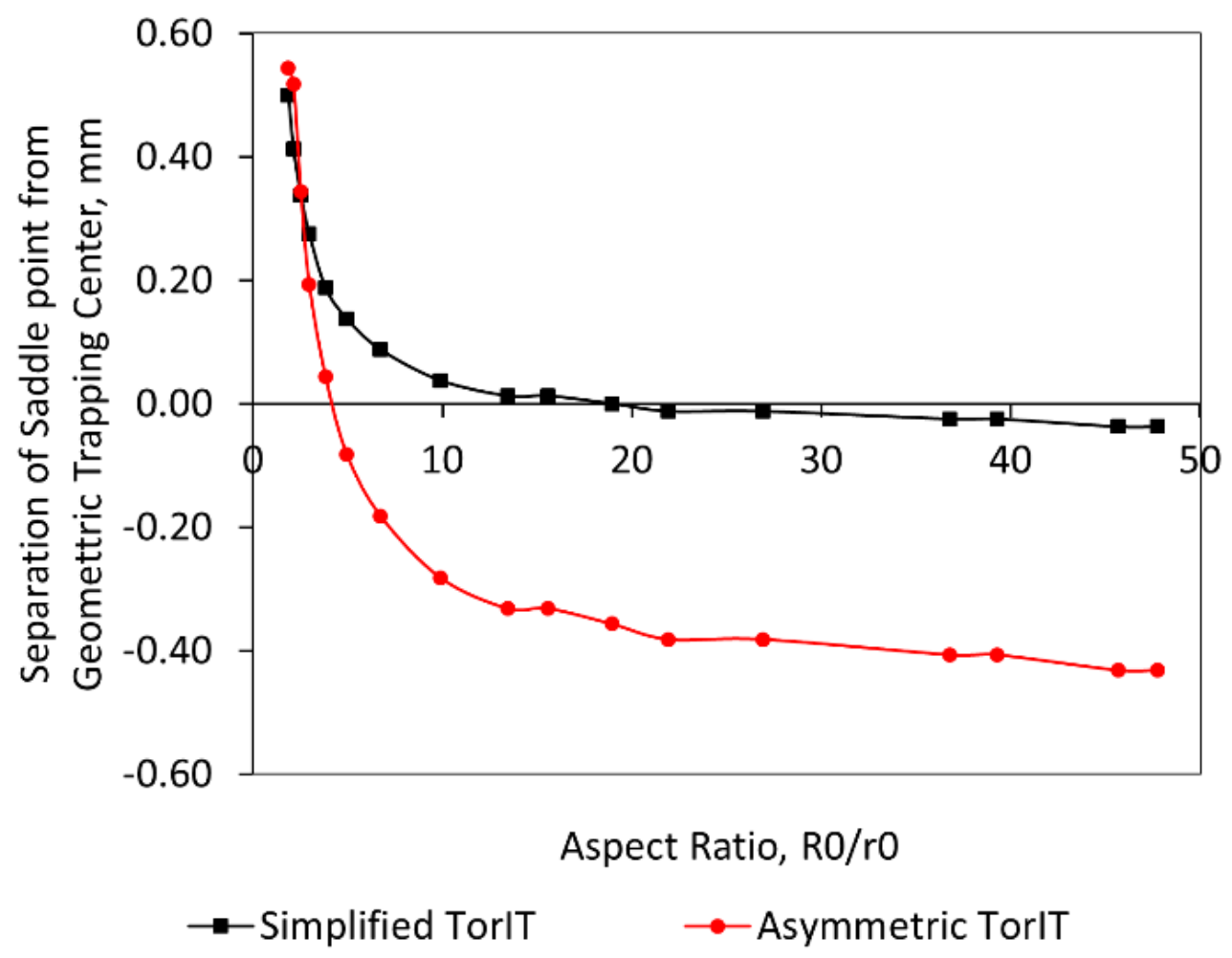

Figure 3: Separation of the saddle point from the geometric trap center in the asymmetric TorIT and simplified TorIT with the aspect ratio.

Fig. 3 shows the separation of saddle points from the geometric trap center respect to AR in asymmetric and simplified TorITs. The, Fig. 4 can be utilized to explain the behavior of TorIT under different ARs. It helps to understand how the asymmetric cross section of asymmetric TorIT (Fig.4(a)) compensates for the increased angular influence of the outer electrode on the Fig.4(b). On the other hand, it explains why the saddle point is not coincided with the geometric center, and also why the saddle point moves when you change aspect ratio while keeping the cross-section the same.

As explained earlier in the discussion, the curvature effect can be acted by the inner and outer ring electrodes. At lower ARs, the trapped ions are pulled towards the inner electrode in the TorIT due to strong coulombic forces acting on the ion cloud by the inner electrode. 
Similarly, at higher ARs, the Coulombic forces exerted by inner and outer electrodes are approximately same. As a result, the ion cloud does not separate from the geometric trapping center. We believe this phenomenon of shifting the saddle point a bit away from the geometric trap center also leads to making the trapping field slightly asymmetric. As explained, when AR tends to infinity, the TorITs tend to become more independent on ARs as the coulombic force from the inner electrode has a negligible effect. However, we predict that the vertical shifting of the saddle points at higher ARs in asymmetric TorIT could arise due to the geometric shape of the electrodes. Because the asymptotes angles of the inner and outer ring asymptotes are not the same which makes permanent shifting of the saddle point from the geometric trapping center even at higher ARs.
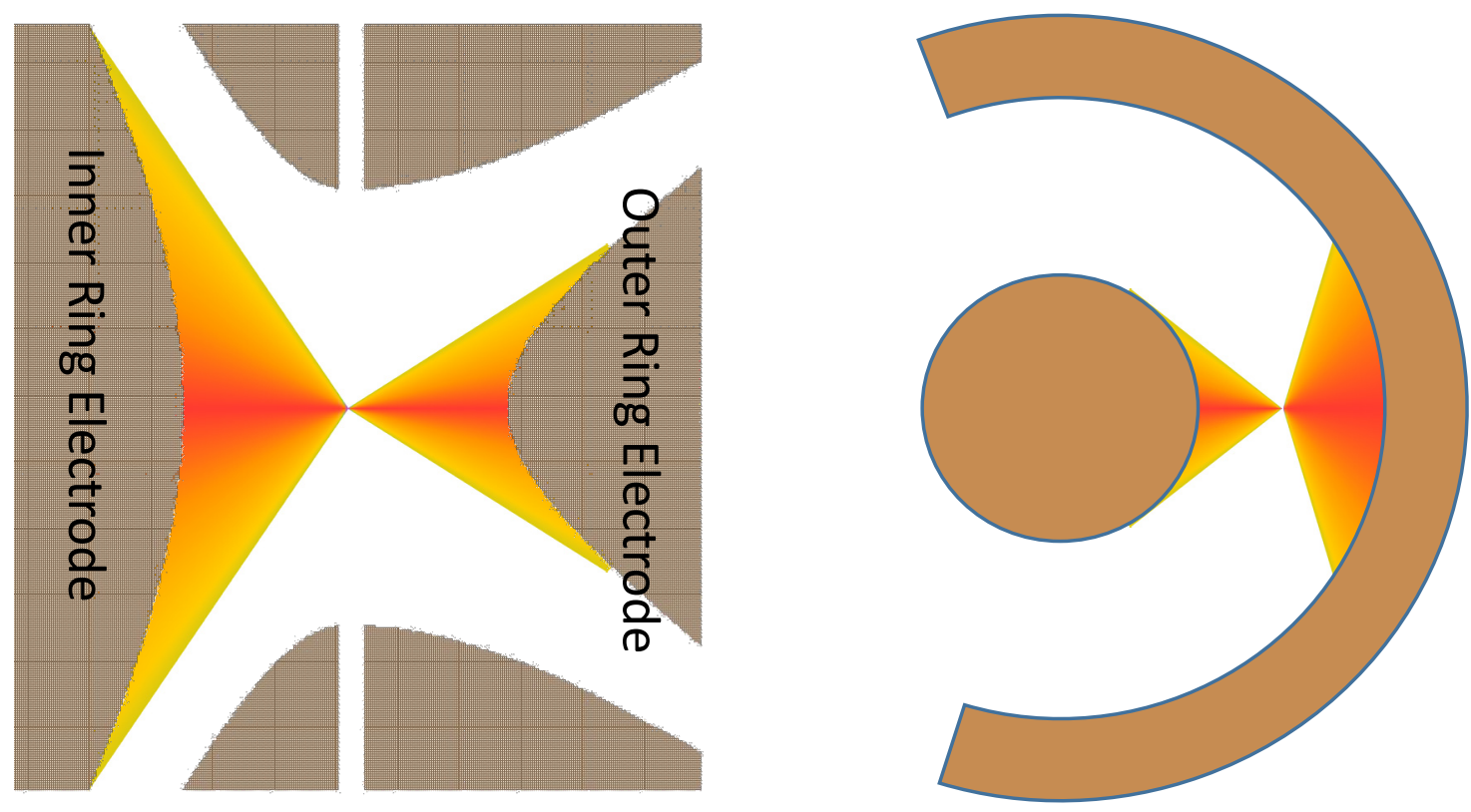

Figure 4: r-z view of the Asymmetric TorIT illustrating the asymmetric Coulombic force on ions resulting in the trapping center being different from the geometric center. The inner electrode exerts strong Columbic force on the ion than the outer electrode due to the asymmetric cross section (a). x-y view of the TorIT with one ion in the ion cloud. The outer electrode exerts strong Columbic force on the ion due to the angular influence of inner and outer ring electrodes (b). 
In QIT ion trap mass spectrometers, the secular frequency can be determined by available mathematical formulas. According to the mathematical formulas, the secular frequency, $\omega_{u}$ is primarily determined by $\beta$, which depends on half the distance between the end caps electrodes of the trap space, $z_{o}, \frac{m}{z}$ of the ion, the radius of the trap, $r_{o}$, r.f. voltage, $V_{0-p}$, DC voltage, and the frequency, $\Omega$, applied to the ring electrode. However, for TorIT, there is no direct method to determine the, $\beta$ and $\omega_{u}$. The topology of the field at $3 \mathrm{D}$ torus at higher AR can be approximately considered as that of a LIT. The LIT (can be considered as a TorIT with infinite AR) has a 2D topology. The topology of the field become a combined version of 3D torus $2 \mathrm{D}$ at higher ARs of TorITs. Hence it might restrict the 3D motion into a $2 \mathrm{D}$ motion.

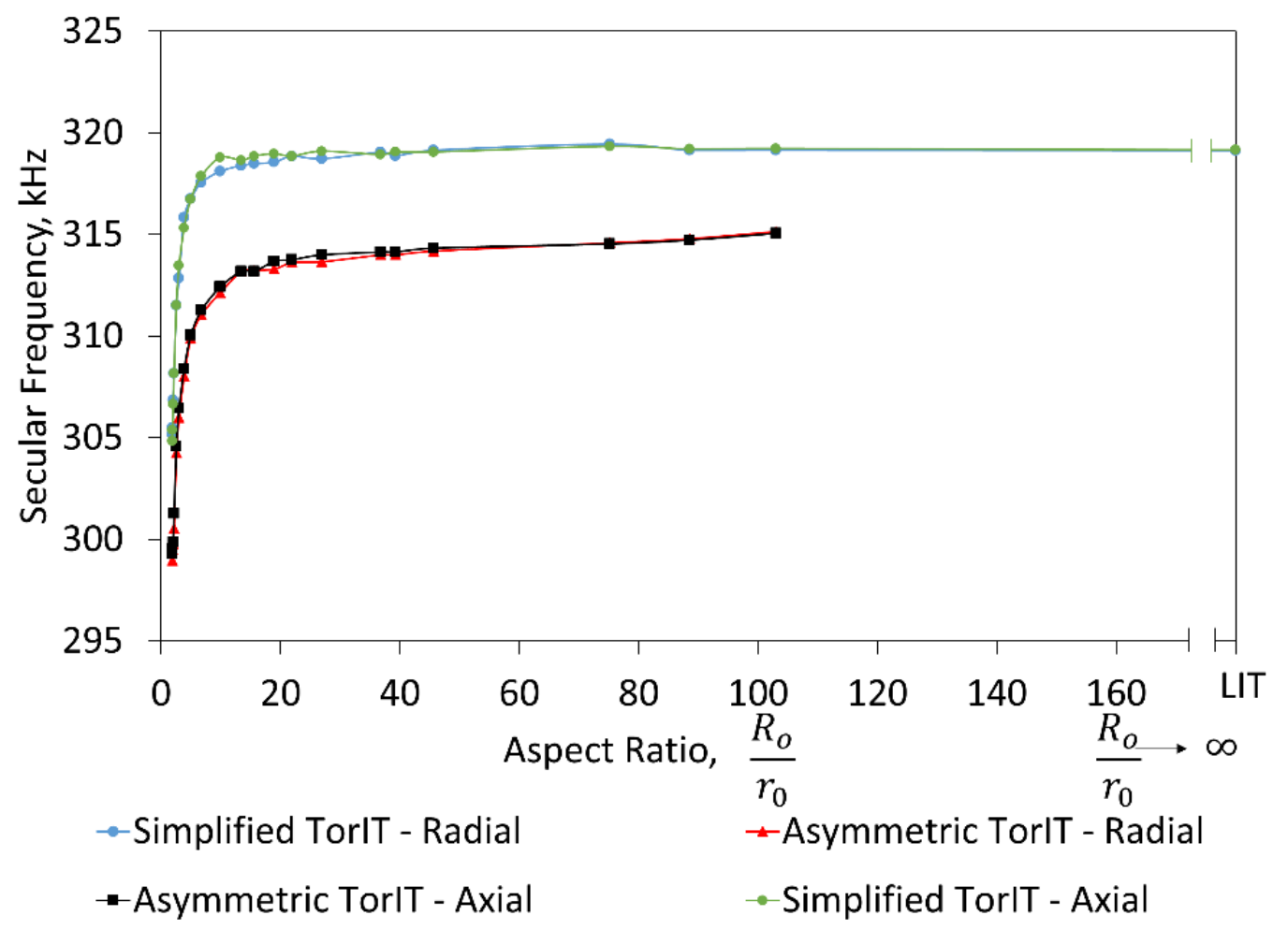

Figure 5: Effect of aspect ratio on the secular frequency of simplified and asymmetric TorITs for an ion of $100 \mathrm{~m} / \mathrm{z}$. The conditions applied to the trap was $500 \mathrm{~V}_{0-\mathrm{p}}$ r.f. voltage at $1 \mathrm{MHz}$ with no DC offset and no collision gas. The last points represent the secular frequencies of a linear-type trap with the electrode cross section corresponding to the toroidal trap. 
Fig. 5 shows how secular frequency changes with AR. Importantly, it follows the same pattern as it does in higher-order variation figures. One reason for the dependency of the secular frequency on the AR can be due to the change in the shape of the trapping potential. Also, the magnitude of the nonlinear electric fields can determine the secular frequency of the ion. As mentioned, the saddle point locates at different places along the radial direction at different ARs. Depending on the position of the saddle point along on the radial direction, the magnitude of nonlinear electric fields changes. This could also attribute to changes of an ion's secular frequency with AR.

The ions in an asymmetric linear ion trap (at infinite AR of asymmetric TorIT) with axial ejection system are unstable. We believe that simplified TorIT does work at higher AR as it was designed with a higher AR in its first design. The simplified electrode geometry was not designed for a specific AR. Also, the nature of the electrode overlaps and the overall radial asymmetry in the simplified TorIT design was much less than that of the asymmetric TorIT. The electrodes geometry of asymmetric TorIT was designed and optimized for a smaller AR. The results conclude that the secular frequency of TorIT is also a function of AR at lower values. This agrees with the Kotana and Mohanty approach about secular frequency calculation of TorITs using numerical simulation [18]. This numerical simulation results prove that an ion in TorITs with same dimensions and different ARs show different secular frequencies. 


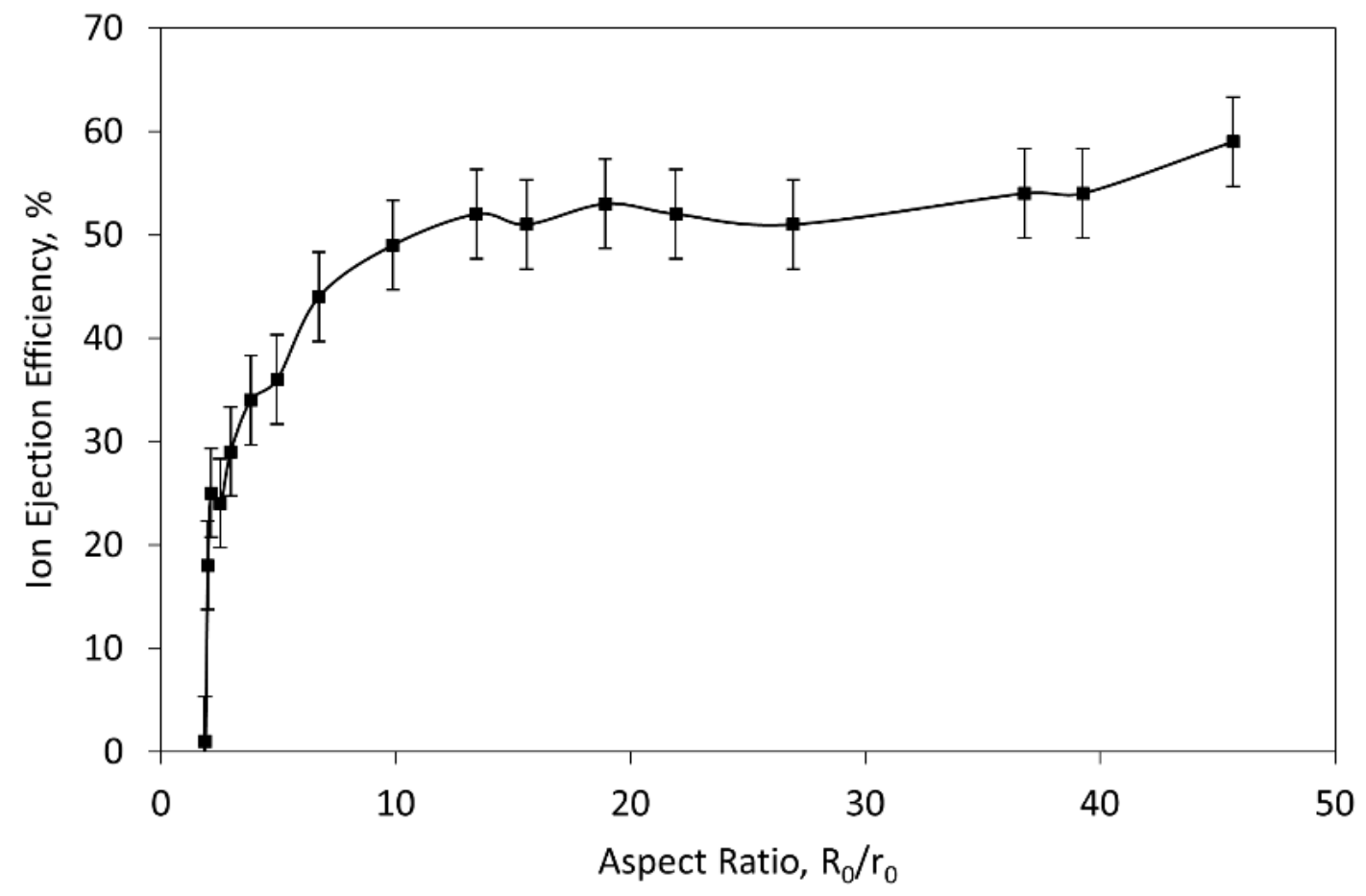

Figure 6: Ion ejection efficiency of simplified TorIT in radial direction. AC voltage applied on the endcap electrode $\left(V_{0-p}=2 \mathrm{~V}\right)$ to excite the ion with $\mathrm{m} / \mathrm{z}$ of 100 via dipolar resonant ejection. The frequency for the $\mathrm{AC}$ voltage was set to a value that is few percent lower the exact secular frequency of the ion. This auxiliary AC frequency was determined experimentally by selecting the maximum frequency that can eject the ions. The hard sphere collision cooling technique was incorporated under the pressure and temperature of $1.5 \mathrm{mTorr}$ and $273 \mathrm{~K}$ respectively.

The ion ejection efficiency of simplified TorIT in the radial direction was investigated with mass selective ejection technique using dipolar resonance. However, introducing the same frequency for the AC auxiliary voltage as the secular frequency caused ions to make a beat motion. This happened in a situation where nonlinear resonance takes place before the dipolar resonance takes place. This can be overcome and eject the ions by applying a dipolar frequency that is lower in small percentage from the exact secular frequency of the ion. However, this frequency may not be the optimum condition for ion ejection. We believe an optimum frequency for the auxiliary ac voltage could enhance the ejection efficiency further. Most of the ions splat on the wall of the slit and the outer ring electrode of the trap. A small positive DC voltage on outer ring electrode and small negative DC voltage on inner electrode can 
minimize the outward ion ejection. According to the Fig. 5, as expected, the ion ejection efficiency follows the same trend as higher-order terms and secular frequency did with aspect ratio. Because, the ejection efficiency is mainly controlled by the higher-order terms present in the trap, hence, as higher orders flatten off with the AR, the effect from the higher-order fields on the ejection efficiency should be the same. Additionally, the dependency of secular frequency on AR has some contribution on the ion ejection efficiency as ions do not eject at the same beta value at different ARs. This effect is very prominant in lower ARs and has a smaller effect after the secular frequency flattens off with AR. However, the ions are ejecting with beta in the 0.6 range, as an exact value for the beta value for TorIT cannot be determined due to the lack of mathematical formulas. Another main observation is the ion trapping efficiency can be neglected or has less significant when AR is lower than two.

\subsection{Conclusion}

A study of Aspect Ratio (AR) in Toroidal Ion Trap (TorIT) is important in optimizing the performance and understanding the physics behind it. This study revealed that the AR of TorIT could induce and superimpose higher-order fields through the curvature effect and reshape the trapping field. The ability to induce superimposed higher-order fields in other extended ion traps such as linear ion traps (LIT) and rectilinear ion traps (RIT) have not been reported before. When the AR tends to infinity the magnitude of superimposed higher-order fields converge to values that a LIT would have with the same electrode geometry. Since the inner and outer electrodes exert two different curvature effects, hence two different columbic forces exert on the ion cloud, the saddle point and the geometric center of the trap do not coincide. Understanding the location of the saddle point is an important parameter in trap design state to locate the position of the exit slits on electrodes. There are no available mathematical formulas that have been derived for the secular frequency of an ion in a TorIT. 
This study provides evidence to reveal the dependency of the secular frequency of an ion with AR. Also, dependency of the secular frequency on AR could also determine other important information such as operating beta value of the ions and ion ejection efficiency. Further, this information can provide a foundation to derive such mathematical formulas. 
2.5 Reference

(1) Austin, D. E.; Wang, M.; Tolley, S. E.; Maas, J. D.; Hawkins, A. R.; Rockwood, A. L.; Tolley, H. D.; Lee, E. D.; Lee, M. L. Halo Ion Trap Mass Spectrometer. Anal. Chem. 2007, 79 (7), 2927-2932.

(2) Badman, E. R.; Cooks, R. G. A Parallel Miniature Cylindrical Ion Trap Array. Anal. Chem. 2000, 72 (14), 3291-3297.

(3) Contreras, J. A.; Murray, J. A.; Tolley, S. E.; Oliphant, J. L.; Tolley, H. D.; Lammert, S. A.; Lee, E. D.; Later, D. W.; Lee, M. L. Hand-Portable Gas Chromatograph-Toroidal Ion Trap Mass Spectrometer (GC-TMS) for Detection of Hazardous Compounds. J. Am. Soc. Mass Spectrom. 2008, 19 (10), 1425-1434.

(4) Lammert, S. A.; Rockwood, A. A.; Wang, M.; Lee, M. L.; Lee, E. D.; Tolley, S. E.; Oliphant, J. R.; Jones, J. L.; Waite, R. W. Miniature Toroidal Radio Frequency Ion Trap Mass Analyzer. J. Am. Soc. Mass Spectrom. 2006, 17 (7), 916-922.

(5) Patterson, G. E.; Guymon, A. J.; Riter, L. S.; Everly, M.; Griep-Raming, J.; Laughlin, B. C.; Ouyang, Z.; Cooks, R. G. Miniature Cylindrical Ion Trap Mass Spectrometer. Anal. Chem. 2002, 74 (24), 6145-6153.

(6) Taylor, N.; Austin, D. E. A Simplified Toroidal Ion Trap Mass Analyzer. Int. J. Mass Spectrom. 2012, 321-322, 25-32.

(7) Wu, Q.; Li, A.; Tian, Y.; Zare, R. N.; Austin, D. E. Miniaturized Linear Wire Ion Trap Mass Analyzer. Anal. Chem. 2016, 88 (15), 7800-7806.

(8) Ouyang, Z.; Wu, G.; Song, Y.; Li, H.; Plass, W. R.; Cooks, R. G. Rectilinear Ion Trap: Concepts, Calculations, and Analytical Performance of a New Mass Analyzer. Anal. Chem. 2004, 76 (16), 4595-4605. 
(9) Gamage, R. W.; Austin, D. E. The Effects of Electrode Misalignments on the Performance of a Miniaturized Linear Wire Ion Trap Mass Spectrometer. Int. J. Mass Spectrom. 2020, 453 (116344), 116344.

(10) Xu, W.; Chappell, W. J.; Cooks, R. G.; Ouyang, Z. Characterization of Electrode Surface Roughness and Its Impact on Ion Trap Mass Analysis. J. Mass Spectrom. 2009, 44 (3), $353-360$.

(11) Peng, Y.; Hansen, B. J.; Quist, H.; Zhang, Z.; Wang, M.; Hawkins, A. R.; Austin, D. E. Coaxial Ion Trap Mass Spectrometer: Concentric Toroidal and Quadrupolar Trapping Regions. Anal. Chem. 2011, 83 (14), 5578-5584.

(12) Lammert, S. A.; Plass, W. R.; Thompson, C. V.; Wise, M. B. Design, Optimization and Initial Performance of a Toroidal Rf Ion Trap Mass Spectrometer. Int. J. Mass Spectrom. 2001, 212 (1-3), 25-40.

(13) Wang, Y.; Zhang, X.; Feng, Y.; Shao, R.; Xiong, X.; Fang, X.; Deng, Y.; Xu, W. Characterization of Geometry Deviation Effects on Ion Trap Mass Analysis: A Comparison Study. Int. J. Mass Spectrom. 2014, 370, 125-131.

(14) Higgs, J. M.; Austin, D. E. Simulations of Ion Motion in Toroidal Ion Traps. Int. J. Mass Spectrom. 2014, 363, 40-51.

(15) Tian, Y.; Higgs, J.; Li, A.; Barney, B.; Austin, D. E. How Far Can Ion Trap Miniaturization Go? Parameter Scaling and Space-Charge Limits for Very Small Cylindrical Ion Traps: Ion Trap Miniaturization. J. Mass Spectrom. 2014, 49 (3), 233240.

(16) Practical Aspects of Ion Trap Mass Spectrometry, Volume I; March, R. E., Todd, J. F. J., Eds.; CRC Press: Boca Raton, FL, 1995. 
(17) Beaty, E. C. Calculated Electrostatic Properties of Ion Traps. Phys. Rev. A Gen. Phys. 1986, 33 (6), 3645-3656.

(18) Kotana, A. N.; Mohanty, A. K. Determination of Multipole Coefficients in Toroidal Ion Trap Mass Analysers. Int. J. Mass Spectrom. 2016, 408, 62-76. 


\section{APPENDIX}

\section{A.1 SIMION 8.0 .lua User Programs}

When simulating Toroidal ion traps in SIMION 8.0, I used user programs to control the voltages to introduce real-life conditions such as neutral ion collision effect and data recording. The user programs were written in .LUA and Comments in .LUA programming is notated with "--" in front. The main user program calls for all the functions including trap initialization, collision, apply r.f. dynamic voltage, and data recording.

\section{Main User Program}

-- Also with hard sphere collisions (see collisions_hs1.lua)

-- After defining the variables, the segments of the program are:

-- trap_initialize: Starting conditions

-- trap_fast_adjust: Voltage control with respect to time

-- trap_tstep_adjust: Controls the time-step size

-- trap_other_actions: Controls collisions, prepares for next time-step

-- trap_terminate: Activates when the ion's flight has ended

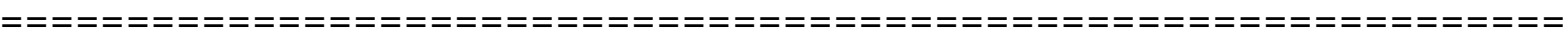
$==ニ=$

simion.workbench_program()

-- adjustable during flight adjustable_DC_voltage $=0.0$ adjustable_RF_amplitude1 $\quad=500$ adjustable_tickle_voltage $\quad=2.0$

-- adjustable in collisions adjustable_mean_free_path_mm $\quad=-1 \quad--$ Mean free path (MFP) (mm) between collisions-- if value of -1 , mean free path is calculated by pressure and other variables below adjustable_gas_mass_amu $\quad=4.0 \quad--$ Mass of background gas particle $(\mathrm{amu})$ adjustable _temperature_k $\quad=273.0$-- Background gas temperature $(\mathrm{K})$ adjustable_pressure_pa adjustable_sigma_m2 adjustable_vx_bar_gas_mmusec adjustable_vy_bar_gas_mmusec adjustable_vz_bar_gas_mmusec adjustable_steps_per_MFP adjustable maxTOF

$=0.3 \quad-$ Background gas pressure $(\mathrm{Pa})$

$=2.27 \mathrm{E}-18$-- Collision-cross section $\left(\mathrm{m}^{\wedge} 2\right)$

$=0 \quad--$ Mean background gas velocity $(\mathrm{mm} / \mathrm{usec})$

$=0 \quad--$ in $x, y, z$ directions

$=0$

$=20.0 \quad--$ Mean number of time steps per MFP

-- tickle parameters

adjustable_tickle_Frequency $\quad=0$

-- voltage control

adjustable phase_angle_deg $\quad=0.0 \quad--$ entry phase angle of ion (deg)

adjustable freqency_hz $\quad=1 \mathrm{E} 6$-- RF frequency of quad in $(\mathrm{hz})$ 
-- display

adjustable pe_update_each_usec $=0.05$-- PE display update time step(usec)

-- collisions

adjustable_mark_collisions

$=0$-- Collision marker flag

adjustable_trace_level

$=0$-- How much trace data (average KE) to output

adjustable_trace_skip

$=100$-- If _trace_level is 2 , this is the number of collisions before

each trace

-- Internal variables

-- Statistics

local ke_averages

local last_collision_times

$=\{\} \quad-$-current running average of KE for each particle

local last_ion_number

$=\{\} \quad-$-last collision time for each particle

$=-1 \quad-$-last known ion number $(-1=$ undefined $)$

local last_speed_ion

$=-1 \quad--$ last known ion speed $(-1=$ undefined $)$

local effective_mean_free_path_mm $=-1$--currently used mean-free path $(-1=$ undefined $)$

local trace_count $\quad=0 \quad-$-count relative to _trace skip

local max_timestep

--maximum time step (usec) that fast_adjust should permit

-- Define constants

local $\mathrm{k}=1.3806505 \mathrm{e}-23$-- Boltzmann constant $(\mathrm{J} / \mathrm{K})$

local $\mathrm{R}=8.3145$-- Ideal gas constant $(\mathrm{J} /(\mathrm{mol} * \mathrm{~K}))$

local kg_amu $=1.6605402 \mathrm{e}-27$-- $(\mathrm{kg} / \mathrm{amu})$ conversion factor

local pi = math.pi -- PI constant

local eV_J $=6.2415095 \mathrm{e}+18--(\mathrm{eV} / \mathrm{J})$ conversion factor

-- Error function (erf)

function $\operatorname{erf}(\mathrm{z})$--erf(z) $=(2 / \mathrm{sqrt}(\mathrm{pi})) *$ integral[0..z] $\exp \left(-\mathrm{t}^{\wedge} 2\right) \mathrm{dt}$

local $z 2=\operatorname{abs}(z)$

local $\mathrm{t}=1 /(1+0.32759109962 * \mathrm{z} 2)$

local res $=(-1.061405429) * \mathrm{t}$

res $=($ res +1.453152027$) * t$

res $=($ res -1.421413741$) * \mathrm{t}$

res $=($ res +0.2844966736$) * t$

res $=(($ res -0.254829592$) * \mathrm{t}) * \exp (-\mathrm{z} 2 * z 2)$

res $=$ res +1

if $z<0$ then res $=-$ res end

return res

end

-- Return a normalized Gaussian random variable (-inf, +inf)

function gaussian_random()

-- Using the Box-Muller algorithm.

local $\mathrm{s}=1$

local v1, v2

while $s>=1$ do

$\mathrm{v} 1=2 *$ rand ()$-1$

$\mathrm{v} 2=2 *$ rand ()$-1$

$\mathrm{s}=\mathrm{v} 1 * \mathrm{v} 1+\mathrm{v} 2 * \mathrm{v} 2$

end

local rand1 $=v 1 * s q r t(-2 * \ln (s) / s)$-- (assume divide by zero improbable?)

return rand1

end

-- Default SIMION initialize segment for ion trap example.

-- This segment is called on every particle creation. 
function trap_initialize()

-- Enable rerun mode (used only for side-effect of disabling trajectory file saving).

sim_rerun_flym $=1$

end

-- Default SIMION fast_adjust segment for ion trap example.

-- This segment is called to modify electrode voltages.

local is_first $\quad=$ true - first call flag

local omega $=1.0--$ frequency ( $\mathrm{rad} / \mathrm{usec})$

local theta $\quad=0.0-$ phase offset (rad)

function segment.fast_adjust() ---- Generate trap RF voltages with fast adjust.-- For efficiency, we calculate some variables only once.

if is_first then

is_first $=$ false

theta $\quad=$ rad(phase_angle_deg)

$=$ freqency_hz $* 2 * 3.14159 * 1 \mathrm{E}-6 \quad--$ frequency $(\mathrm{rad} / \mathrm{usec})$

omega

end

if ion_time_of_flight $<=1 \mathrm{e} 3$ then

adj_elect01 = _DC_voltage + _RF_amplitude1 $* \sin ($ theta +ion_time_of_flight $*$ omega)

adj_elect02 = _DC_voltage $--\mathrm{U}+\mathrm{V}^{*} \sin \left(\right.$ theta $+\mathrm{t}^{*}$ omega)

adj_elect03 $=$ _DC_voltage + _RF_amplitude $1 * \sin ($ theta +ion_time_of_flight $*$ omega)

adj_elect04 $=0$

else

tickle $=\_$tickle_voltage $* \sin ($ _tickle_Frequency $* 2 * 3.14159 * 1 \mathrm{E}-6 *$ ion_time_of_flight)

adj_elect01 $=$ _DC_voltage + _RF_amplitude $1 * \sin ($ theta + ion_time_of_flight $*$ omega $)$

adj_elect02 $=$ _DC_voltage + tickle

adj_elect03 $=$ _DC_voltage + _RF_amplitude $1 * \sin ($ theta + ion_time_of_flight $*$ omega $)$

adj_elect04 $=0$

--print(ion_time_of_flight .. ',' .. adj_elect05 .. ',' ..adj_elect04..',' )

end

end

-- Default SIMION time_step adjust segment for ion trap example.

-- This segment is called to override time-step size.

function segment.tstep_adjust()-- Keep time step $<=0.1$ usec.

ion_time_step $=\min ($ ion_time_step, 0.1 ) $\quad-$ - X usec

end

-- Default SIMION other_actions segment for ion trap example.

-- This segment is called on every time-step.

function segment.other_actions()

if _pressure_pa $==0$ then -- collisions disabled

return

end

local vx = ion_vx_mm - _vx_bar_gas_mmusec

local vy = ion_vy_mm - _vy_bar_gas_mmusec 
local vz = ion_vz_mm - _vz_bar_gas_mmusec

local speed_ion $=\operatorname{sqrt}\left(v^{\wedge} 2+v y^{\wedge} 2+v z^{\wedge} 2\right)$

if speed_ion $<1 E-7$ then

speed_ion $=1 \mathrm{E}-7$

-- prevent divide by zero and such effects later on

end

if _mean_free_path_mm $>0$ then

-- explicitly specified

effective_mean_free_path_mm = _mean_free_path_mm

else

if last_ion_number $\sim=$ ion_number or abs(speed_ion / last_speed_ion - 1) $>0.05$-- changed

then

local c_bar_gas $=\operatorname{sqrt}\left(8^{*} \mathrm{k}^{*}\right.$ _temperature_k/pi/(_gas_mass_amu $\left.\left.* \mathrm{~kg} \_a m u\right)\right) / 1000$

local c_star_gas $=\operatorname{sqrt}\left(2 * k^{*}\right.$ _temperature_k/(_gas_mass_amu $*$ kg_amu $\left.)\right) / 1000$

local $\mathrm{s}=$ speed_ion $/$ c_star_gas

local c_bar_rel = c_bar_gas $*((\mathrm{~s}+1 /(2 * \mathrm{~s})) * 0.5 * \operatorname{sqrt}(\mathrm{pi}) * \operatorname{erf}(\mathrm{s})+0.5 * \exp (-\mathrm{s} * \mathrm{~s}))$

effective_mean_free_path_mm $=1000 * 4 *(\mathrm{k} *$ _temperature_k $*($ speed_ion / c_bar_rel $)$ /

(_pressure_pa*_sigma_m2))

last_speed_ion = speed_ion

last_ion_number = ion_number

end

end

max_timestep $=$ effective_mean_free_path_mm / speed_ion / _steps_per_MFP

local collision_prob $=1$ -

exp(- speed_ion * ion_time_step / effective_mean_free_path_mm)

if rand ()$>$ collision_prob then

return -- no collision

end

----- Handle collision.

local vr_stdev_gas $=\operatorname{sqrt}(\mathrm{k} *$ _temperature_k / (_gas_mass_amu $*$ kg_amu)) $/ 1000$

local vx_gas, vy_gas, vz_gas -- computed velocities

local scale $=$ speed_ion + vr_stdev_gas $* 1.732 * 3$--sqrt(3) $=\sim 1.732$

repeat

vx_gas $=$ gaussian_random ()$* v r \_s t d e v \_g a s$

vy_gas $=$ gaussian_random ()$* v r \_s t d e v \_g a s$

vz_gas $=$ gaussian_random ()$* v v_{-}$stdev_gas

local len $=\operatorname{sqrt}\left(\left(v x \_g a s-v x\right)^{\wedge} 2+\left(v y \_g a s-v y\right)^{\wedge} 2+(v z \text { gas }-v z)^{\wedge} 2\right)$

until rand ()$<$ len $/$ scale

$\mathrm{vx}=\mathrm{vx}-\mathrm{vx} \_\mathrm{gas}$

$v y=v y-v y \_g a s$

$\mathrm{vz}=\mathrm{vz}-\mathrm{vz} \_\mathrm{gas}$

local impact_offset $=\operatorname{sqrt}(0.999999999 *$ rand ()$)$

local impact_angle $=$ asin(impact_offset)

local impact_theta $=2 *$ pi*rand $($ )

local speed_ion_r, az_ion_r, el_ion_r = rect3d_to_polar3d(vx, vy, vz)

local vr_ion $=$ speed_ion_r $* \cos ($ impact_angle $)$-- radial velocity

local vt_ion $=$ speed_ion_r $*$ sin(impact_angle) - - normal velocity

local vr_ion $2=\left(v r \_i o n *\left(i o n \_m a s s-\right.\right.$ _gas_mass_amu $\left.)\right) /($ ion_mass + _gas_mass_amu $)$

$v x, v y, v z=$ elevation_rotate(90 - deg(impact_angle), vr_ion2, vt_ion, 0)

$v x, v y, v z=$ azimuth_rotate(deg(impact_theta), vx, vy, vz)

$v x, v y, v z=$ elevation_rotate $(-90+$ el_ion_r, vx, vy, vz)

$v x, v y, v z=$ azimuth_rotate(az_ion_r, vx, vy, vz)

$v x=v x+v x \_g a s+$ _vx_bar_gas_mmusec

$v y=v y+v y \_g a s+$ _vy_bar_gas_mmusec

$\mathrm{vz}=\mathrm{vz}+\mathrm{vz} \_$gas + _vz_bar_gas_mmusec 
if _trace_level $>=1$ then

--Compute new ion speed and KE

local speed_ion $2=\operatorname{sqrt}($ ion_vx_mm^2 + ion_vy_mm^2 + ion_vz_mm^2)

local ke2_ion = speed_to_ke(speed_ion2, ion_mass)

local dt = ion_time_of_flight - (last_collision_times[ion_number] or 0 )

reset_time $=$ ion_time_of_flight $* 0.5$

local $\mathrm{w}=1$ - (dt / reset_time) $--\sim=\exp (-\mathrm{dt} /$ reset_time)

ke_averages[ion_number] $=\mathrm{w} *($ ke_averages[ion_number] or ke2_ion $)+(1-\mathrm{w}) *$ ke2_ion

if _trace_level $>=2$ then -- more detail

local T_ion $=$ ke_averages[ion_number] $/ \mathrm{eV}$ ] $/(1.5 * \mathrm{k})$

if trace_count \% _trace_skip $==0$ then

print(string.format( $" \mathrm{n}=, \% \mathrm{~d}, \mathrm{TOF}=, \% 0.3 \mathrm{~g}$,ion $\mathrm{KE}(\mathrm{eV})=, \% 0.3 e$,ion mean $\mathrm{KE}(\mathrm{eV})=, " . . \% 0.3 e$,ion

mean temp $(\mathrm{K})=, \% 0.3 \mathrm{e} "$,ion_number, ion_time_of_flight, ke2_ion,ke_averages[ion_number], $\mathrm{T}$ _ion))

end

trace_count $=($ trace_count +1$) \%$ trace_skip

end

last_collision_times[ion_number] = ion_time_of_flight

end

if _mark_collisions $\sim=0$ then

mark()

-- draw dot at collision point

end

local next_pe_update $=0.0$

if abs(ion_time_of_flight - pe_update_each_usec) $>=$ pe_update_each_usec then

pe_update_each_usec $=$ ion_time_of_flight

sim_update_pe_surface $=1$ end

end

function segment. Terminate()

if _trace_level $>=1$ then

-- ion temperature

local T_ion $=$ ke_averages[ion_number] $/ \mathrm{eV} \_\mathrm{J} /(1.5 * \mathrm{k})$

print(string.format(" $n=, \% d, T O F=, \% 0.3 g$,ion mean $\mathrm{KE}(\mathrm{eV})=, \% 0.3 e$,ion mean $\operatorname{temp}(\mathrm{K})=, \% 0.3 \mathrm{e} "$, ion_number, ion_time_of_flight, ke_averages[ion_number], T_ion))

end

-- Disable rerun mode from initialize segment (we don't really want to rerun)

sim_rerun_flym $=0$

end

-- Install default SIMION segments for ion trap example.

-- This provides a quick method for programs that use this

-- file to install the default ion trap functionality.

function trap_install_segments()

segment.initialize $=$ trap_initialize

segment.fast_adjust $=$ trap_fast_adjust

segment.tstep_adjust = trap_tstep_adjust

segment.other_actions $=$ trap_other_actions

segment.terminate $=$ trap_terminate

end 
A user program was introduced to the function segment. Terminate () To calculate the amount ions can be ejected from the trap for the ion ejection efficiency figure. Also, the accuracy of this result was cross-checked by exporting the splat position into a .csv file by the SIMION.

\section{A.2 Data Recording}

function segment. Terminate()

local count $=0$

outfile $=$ 'file_name.csv'

fout=io.open(outfile,'a')

local $r=\operatorname{sqrt}\left((\text { ion_py_mm })^{\wedge} 2+(\text { ion_pz_mm })^{\wedge} 2\right)$

if $r<100.800$

then

count $=$ count +1

fout:write(count..','..ion_number..',')

fout:write('\n')

end

fout:close()

end

\section{A.2 MATLAB Scripts for Data Processing}

I used to calculate contribution of higher-order terms, the electric field profile inside the ion trap along the radial and axial directions and the secular frequencies of ions using MATLAB scripts. Comments in MATLAB scripts are notated with "\%" in front.

\section{A.2.1 MATLAB Script to Calculate Higher-Order Terms}

The magnitude of higher-order terms in axial and radial directions in TorIT can be investigated by using a Fast Fourier transform function (FFT) in MATLAB. The data collected included the voltage and the ion's position in radial (y) and axial (x) directions. This script was designed to read in the position and corresponding position of the ion. Then the data is fitted into $8^{\text {th }}$ order polynomial function using polyfit function to calculate the voltage profile. Then the coefficients of the each higher-order terms are represented relative to the quadrupole term. Finally, polyder function is used to plot the electric field profiles. 
$\mathrm{D}=$ dlmread('C: $\mid$ Users $\backslash$ Praneeth $\backslash$ Desktop $\backslash$ New $\backslash$ New folder (2)\Trap.csv'); \% Read the file xpos $=\mathrm{D}(1:$ end, 1$) ; \%$ Read for position

$y$ vol $=\mathrm{D}(1:$ end, 2$) ; \%$ Read for voltage at each point

$\operatorname{vmax}=\max (y \mathrm{vol}) ; \%$ find the maximum voltage

xmax_index $=$ find $(y v o l==v \max ) ; \%$ find the index where voltage get maximum

$x \max =x \operatorname{xpos}\left(x \max \_\right.$index $) ; \%$ coordinate at voltage get maximum

Oxpos $=$ xpos-xmax; $\%$ represent data relative to the saddle point

Nxpos = Oxpos;

NxposIl = xmax_index-150; \% find 150 data points relative to one side of the saddle point

Nxposul=xmax_index+150; \% find 150 data points relative to the other side of the saddle point

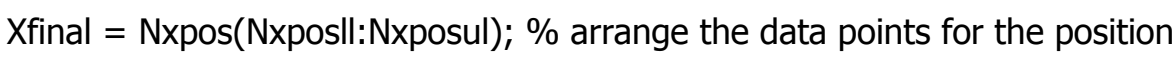

Yfinal = yvol(Nxposll:Nxposul); \% arrange the data points for the voltage

Percentage $=\operatorname{abs}(x f i n a l(1) * 100)$;

$\mathrm{pf}=$ polyfit(xfinal,yfinal,8); \% fit the position into a $8^{\text {th }}$ order polynomial function to get the voltage profile.

fraction $=(\mathrm{pf} / \mathrm{pf}(1,7) * 100) ; \%$ Find the higher-order relative to the quadrupole term.

$\mathrm{E}=\operatorname{polyder(pf);\% Calculate}$ the electric field.

Filename='C: $\mid$ Users $\backslash$ Praneeth $\backslash$ Desktop $\backslash N e w \backslash N e w$ folder (2)\Analysis_Trap.xlsx';

Sheet1 $=1$;

Sheet2=2;

Sheet3=3;

xIRange1='A1';

xIRange2='A1';

xIRange3='A1';

xlswrite(Filename,pf,Sheet1,xIRange1)

xlswrite(Filename,fraction,Sheet2,xIRange2)

xlswrite(Filename,E,Sheet3,xIRange3)

\section{A.2.3 MATLAB Script to generate frequency spectra}

The frequency spectrums in axial and radial directions of the ion's motion in TorIT can be investigated by using a Fast Fourier transform function (FFT) in MATLAB. The data collected included the time $(0.01 \mu \mathrm{S}$ evenly spaced) and the ion's position in radial (y) and axial (x) directions. This script was designed to read in the time and position of the ion, eliminate duplicates, center the average at zero and perform the Fourier transform, and identify frequencies of the ion motion in $\mathrm{Hz}$. 
$D=$ dlmread('H:\Neutral Fly $\backslash$ New folder $\backslash 12 \backslash$ Twele.csv'); \% location to read the file $\mathrm{x}=\mathrm{D}(1$ :end, 2$) ; \%$ read the position along the axial direction

$y=D(1:$ end, 3$) ; \%$ read the position along the radial direction

$\mathrm{t} 1=\mathrm{D}(1$ :end, 1$) ; \%$ read the time at each positions

$\mathrm{t}=\mathrm{t} 1 * 1 \mathrm{e}-6 ; \%$ convert the micro seconds in to mili seconds

$\%$ eliminate the duplicates

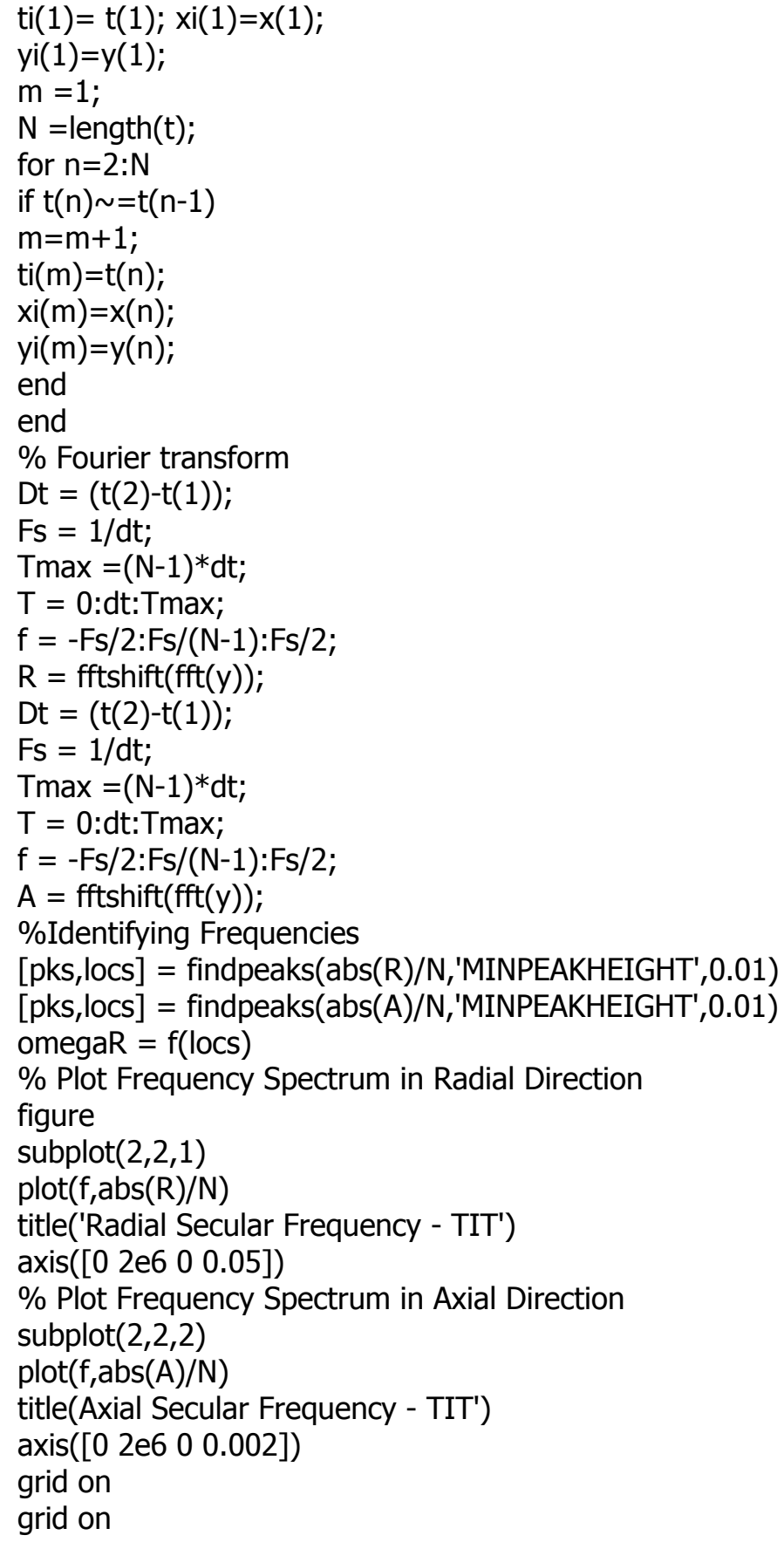

